\title{
Non-thermal dark matter production from the electroweak phase transition: multi-TeV WIMPs and "baby-zillas"
}

Adam Falkowski ${ }^{a}$ and Jose M. No ${ }^{b, c}$

${ }^{a}$ Laboratoire de Physique Théorique d'Orsay, UMR8627-CNRS, Université Paris-Sud, 91405 Orsay, France

${ }^{b}$ Service de Physique Théorique, Université Libre de Bruxelles, B-1050 Bruxelles, Belgium

${ }^{c}$ University of Sussex, Department of Physics and Astronomy, BN1 9QH Brighton, United Kingdom

E-mail: adam.falkowski@th.u-psud.fr, J.M.No@sussex.ac.uk

ABSTRACT: Particle production at the end of a first-order electroweak phase transition may be rather generic in theories beyond the standard model. Dark matter may then be abundantly produced by this mechanism if it has a sizable coupling to the Higgs field. For an electroweak phase transition occuring at a temperature $T_{\mathrm{EW}} \sim 50-100 \mathrm{GeV}$, nonthermally generated dark matter with mass $M_{X}>\mathrm{TeV}$ will survive thermalization after the phase transition, and could then potentially account for the observed dark matter relic density in scenarios where a thermal dark matter component is either too small or absent. Dark matter in these scenarios could then either be multi-TeV WIMPs whose relic abundace is mostly generated at the electroweak phase transition, or "Baby-Zillas" with mass $M_{\mathrm{GUT}} \gg M_{X} \gg v_{\mathrm{EW}}$ that never reach thermal equilibrium in the early universe.

Keywords: Higgs Physics, Beyond Standard Model, Cosmology of Theories beyond the SM

ARXIV EPRINT: 1211.5615 


\section{Contents}

1 Introduction $\quad 1$

2 Particle production at the EW phase transition 3

2.1 Bubble collisions in the EW phase transition 3

2.2 Particle production through bubble collisions 8

3 Particle production via the Higgs portal $\quad 12$

$\begin{array}{lll}3.1 & \text { Scalars } & 12\end{array}$

$\begin{array}{lll}3.2 & \text { Fermions } & 13\end{array}$

$\begin{array}{lll}3.3 & \text { Vector bosons } & 15\end{array}$

$\begin{array}{lll}3.4 & \text { Backreaction and relative efficiency } & 17\end{array}$

4 Non-thermal multi-TeV WIMP dark matter $\quad 19$

$\begin{array}{ll}4.1 \text { Higgs-vector dark matter interplay } & 19\end{array}$

4.2 Fate of non-thermally produced vector dark matter 21

5 Baby-zillas: super-heavy dark matter from the EW phase transition $\quad 23$

5.1 Bounds on the reheating temperature after inflation 24

6 Conclusions $\quad 25$

A Asymmetric dark matter production $\quad \mathbf{2 6}$

A.1 Decay asymmetries: producing a dark matter asymmetry 26

$\begin{array}{lll}\text { A.2 } & \text { Fate of the generated asymmetric abundance } & 28\end{array}$

\section{Introduction}

The most popular paradigm for the origin of dark matter (DM) in the Universe is the thermal freeze-out. In that scenario, the dark matter particle with mass $M_{X}$ annihilates into matter with a cross section $\langle\sigma v\rangle_{\text {thermal }} \sim 3 \times 10^{-26} \mathrm{~cm}^{3} / \mathrm{s}$. This ensures dark matter is in thermal equilibrium with the rest of the plasma in the early universe while $T \gtrsim$ $M$ but decouples when $T \sim M_{X} / 20$, leaving the relic abundance in agreement with the value $\Omega_{X}=0.228 \pm 0.027$ measured by WMAP [1]. Incidentally, $\langle\sigma v\rangle_{\text {thermal }}$ is a generic cross section for a weak scale mass particle interacting with order one couplings, this fact being referred to as the WIMP miracle. In spite of these attractive features, non-thermal mechanisms of dark matter production have also received considerable attention. Examples include right-handed neutrinos produced by oscillations [2], axions produced by vacuum misalignment [3-5], winos produced from moduli decays [6], and super-massive dark matter (WIMP-zillas) produced during reheating after inflation [7-10]. These studies allow one to 
recognize a wider range of possible collider and astrophysical signals of dark matter than what would result from the thermal WIMP scenario.

In this paper we study the possibility of non-thermal dark matter production during a first-order electroweak (EW) phase transition. Bubble collisions at the end of the EW phase transition may give rise to abundant non-thermal particle production when a sizable amount of the energy budget of the transition is stored in the bubble walls, possibly leading to new and appealing scenarios. Many models of dark matter contain a direct coupling between the Higgs and the dark matter candidate fields (MSSM and its extensions, Little Higgs theories with T-parity and DM extensions of the standard model (SM) via the Higgs portal, to name a few). It is thus reasonable to expect that dark matter may be abundantly produced non-thermally at the end of a first-order EW phase transition. Note that, much like in the thermal WIMP case, dark matter would then be a particle with $M_{X} \sim 10 \mathrm{GeV}-10 \mathrm{TeV}$ with significant coupling to the SM, thus being within reach of colliders and DM direct detection experiments.

There is however one generic problem with this scenario. Since the temperature of the Universe right after the EW phase transition is $T_{\mathrm{EW}} \sim 50-100 \mathrm{GeV}$ (for strong transitions $T_{\mathrm{EW}}$ may be somewhat lower than $100 \mathrm{GeV}$ ), thermalization will typically lead to a washout of the non-thermal abundance, thus rendering the particle production at the EW phase transition irrelevant for the subsequent evolution of the Universe. The wash-out process can nevertheless be avoided in a number of ways, each resulting in a scenario where non-thermal dark matter production is (fully or partially) responsible for the observed dark matter relic density. One possibility, recently outlined in [11], is to allow for a few e-foldings of inflation prior to the beginning of the transition (which can happen for very strong EW phase transitions), diluting the plasma and leaving the Universe partially empty. If the reheating temperature after the phase transition is low, $T_{\mathrm{RH}} \ll 100 \mathrm{GeV}$, it may be possible for a dark matter candidate with weak couplings to the Higgs field and mass $M_{X} \sim 100 \mathrm{GeV}$ to remain out of thermal equilibrium after the EW phase transition. In this paper we investigate other scenarios allowing for a survival of the non-thermal abundance.

One possibility corresponds to the case of relatively heavy (multi-TeV) dark matter: for $M_{X} \gtrsim 1 \mathrm{TeV}$, dark matter will be very non-relativistic when the EW phase transition takes place, and the decoupling/freeze-out temperature $T_{\mathrm{FO}}$ will satisfy $T_{\mathrm{FO}} \sim M_{X} / 20 \gtrsim T_{\mathrm{EW}}$. Then, heavy dark matter produced non-thermally through bubble collisions may remain out of thermal equilibrium after the EW phase transition (or at least wash-out will be partially avoided). Another possibility is that bubble collisions produce super-heavy dark matter, ${ }^{1}$ $M_{X} \sim 10^{6}-10^{8} \mathrm{GeV}$, a scenario we call "baby-zillas". We argue this may be possible for a very strong EW phase transition and dark matter having a large coupling the Higgs. In order for baby-zillas with $M_{X} \gg v_{\text {EW }}$ to be a viable dark matter candidate, they must have never reached thermal equilibrium in the early universe after inflation, since otherwise they would have over-closed the universe. This sets a relatively low upper bound on the reheating temperature after inflation in that scenario. Finally, asymmetric dark matter

\footnotetext{
${ }^{1}$ Very heavy dark matter production via bubble collisions at the end of a first-order phase transition was discussed earlier [7] in the context of inflation, the inflaton being the field undergoing the phase transition.
} 
production might allow to avoid complete wash-out of the non-thermal abundance through thermalization after the EW phase transition.

The paper is organized as follows: in section 2 we review the formalism that describes particle production at the end of the EW phase transition for the case of very elastic bubble collisions $[12,13]$ and extend it to the case of very inelastic ones, highlighting the differences between both scenarios [14]. Then, in section 3 we explicitly compute the particle production efficiency of scalar, fermion, and vector boson particles coupled to the Higgs (either directly or via an effective Higgs portal). In sections 4 and 5 we focus on dark matter production at the end of the EW phase transition. First we discuss in section 4 the conditions for non-thermally produced dark matter to avoid subsequent wash-out and constitute the bulk of the present dark matter density, selecting heavy (multi-TeV) vector boson dark matter as a viable example. We go on to analyze in detail non-thermal dark matter production in that scenario and the subsequent evolution of the non-thermally generated abundance after the EW phase transition, including finally a discussion on the current XENON100 bounds and direct detection prospects. Then, in section 5 we study the non-thermal production of very heavy $\left(M_{X} \gg v_{\mathrm{EW}}\right)$ vector boson dark matter, and outline the conditions under which these baby-zillas constitute a viable dark matter candidate. In the case of asymmetric non-thermal dark matter production, we find it difficult to avoid subsequent wash-out, and the discussion is left for an appendix. We summarize our results and conclude in section 6 .

\section{Particle production at the EW phase transition}

\subsection{Bubble collisions in the EW phase transition}

If the early Universe was hotter than $T_{\mathrm{EW}} \sim 100 \mathrm{GeV}$ it must have undergone an EW phase transition at some point in its history. Within the SM, the EW phase transition is a smooth cross-over, however it is conceivable that new degrees of freedom beyond the SM modify the Higgs potential so as to make the transition first order. This is what we assume throughout this paper, without specifying the full theory that makes the first order transition possible. In that case, the EW phase transition proceeded through nucleation and expansion of bubbles of true Higgs vacuum, which eventually collided among each other completing the transition. As this was happening during the radiation dominated era, the bubble expansion process would then have taken place in a thermal environment (except for the case when a period of inflation would have preceded the phase transition).

For a first order phase transition occuring in a thermal environment, the study of the bubble expansion process reveals that the thermal plasma exerts some amount of friction on the expanding bubble wall, and this friction tends to balance the pressure difference on the bubble wall driving the expansion. In the usual picture, nucleated bubbles reach a stationary state after a very short period of acceleration, with a constant wall velocity depending specifically on the interactions of the bubble wall with the degrees of freedom in the plasma [15-19] and on the resulting fluid dynamics [20-25] (see [26] for a review). In this case, the amount of energy stored in the bubble walls at the time of the bubble 
collisions is negligible compared to the available energy of the transition, since most of this available energy gets converted into plasma bulk motion and thermal energy [27].

However, this picture was recently challenged in [28], where it was shown that the friction exerted by the plasma may saturate to a finite value for ultrarelativistic bubble walls. As a consequence the stationary state assumption will no longer be true when the pressure difference on the bubble wall is larger than the friction saturation value, which may happen for strongly first order phase transitions. In this scenario, if there are no hydrodynamic obstacles that prohibit the bubble walls to become highly relativistic in the first place (see however [29]), bubbles will expand in an accelerated way ("the so-called runaway bubbles), with almost all the energy of the transition being used to accelerate the bubble walls ${ }^{2}[26]$. By the end of the phase transition (when bubbles start colliding), these runaway bubbles may reach very large values of $\gamma_{w}$ :

$$
\gamma_{w} \lesssim \gamma_{w}^{\max } \sim \frac{\beta^{-1}}{H^{-1}} \frac{M_{\mathrm{pl}}}{v_{T}} \sim 10^{15}
$$

with $v_{T}$ the value of the Higgs VEV in the broken phase and $\beta^{-1} \sim\left(10^{-3}-10^{-2}\right) H^{-1}$ being the duration of the phase transition [30]. The estimate (2.1) follows from balancing the surface energy on the bubble wall (2.5) and the energy available inside the bubble.

Once bubbles start colliding, the energy stored on the bubble walls will be liberated into the plasma. As argued above, for "runaway" bubbles this will correspond to a very large portion of the energy budget of the phase transition, and therefore this process can be very important. Under certain circumstances, this may also hold true for highly relativistic bubble walls $\left(\gamma_{w} \gg 1\right)$ that reach a stationary state long before bubble collisions start (meaning that $\gamma_{w} \ll \gamma_{w}^{\max }$ ), in which case the amount of energy stored in the bubble walls will be very small compared to the available energy of the transition, but still important when released into the plasma at the end of the transition.

The process of bubble collisions in cosmological first order phase transitions is by itself a very complicated one. Consider a configuration of two planar bubble walls ${ }^{3}$ initially far away from each other, that approach and collide [12, 13, 31]. Depending of the shape of the potential for the scalar field $\phi$ driving the transition (in our case, the Higgs field $h$ ), the bubble collision will be approximately elastic or partially inelastic [12, 13] (see also [14]). In the first case, the walls reflect off one another after the collision, which reestablishes a region of symmetric phase between the bubble walls. For a perfectly elastic collision the field profile of the colliding walls in the limit of infinitely thin bubble walls (taken as

\footnotetext{
${ }^{2}$ This situation may also arise if, under very specific circumstances, a few e-foldings of inflation are achieved prior to the beginning of the EW phase transition (see [11] for a natural realization of this scenario), diluting the plasma and leaving the Universe mostly empty. In this case the expansion of the bubbles effectively takes place in vacuum, and the nucleated bubbles expand in an accelerated way due to the absence of friction.

${ }^{3}$ At the time of the collision, the bubbles are so large compared to the relevant microscopical scales, that their walls may be considered planar as a good approximation.
} 
step-functions) can be written as [13]

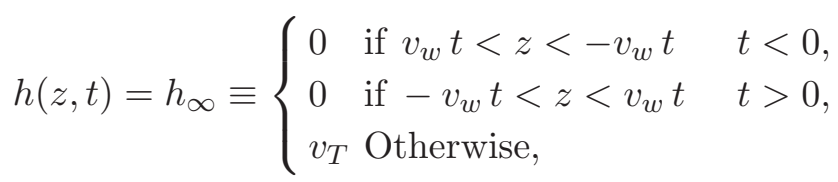

where $v_{w}$ is the bubble wall velocity, the bubble walls move in the $z$-direction and the collision is assumed to happen at $t=0$. Since we are ultimately interested in scenarios where $\gamma_{w} \gg 1$, we will take the ultrarelativistic limit $v_{w} \rightarrow 1$ in the rest of the section. The field profile (2.2) neglects the thickness of the bubble walls $l_{w}$ (generically, $l_{w} \sim$ $(10-30) / T_{\mathrm{EW}}$, with $\left.T_{\mathrm{EW}} \sim 50-100 \mathrm{GeV}\right)$. To capture the wall thickness effects one can consider another ansatz for the profile of the colliding bubble walls:

$$
\begin{aligned}
h(z, t) & =h_{l_{w}} \equiv \frac{v_{T}}{2}\left[\operatorname{Tanh}\left(\gamma_{w} \frac{t+|z|}{l_{w}}\right)-\operatorname{Tanh}\left(\gamma_{w} \frac{t-|z|}{l_{w}}\right)\right] \\
& =\frac{v_{T}}{2}\left[2+\operatorname{Tanh}\left(\gamma_{w} \frac{z-|t|}{l_{w}}\right)-\operatorname{Tanh}\left(\gamma_{w} \frac{z+|t|}{l_{w}}\right)\right] .
\end{aligned}
$$

A perfectly elastic collision is however an idealized situation, as one expects a certain degree of inelasticity in a realistic collision. Moreover, even for a very elastic collision the bubble walls will eventually be drawn back together by vacuum pressure and collide again. A quantitative picture of the collision of two planar bubble walls can be obtained by studying the evolution equation for the scalar field configuration $h(z, t)$ subject to the potential $V(h)$ :

$$
\left(\partial_{t}^{2}-\partial_{z}^{2}\right) h(z, t)=-\frac{\partial V(h)}{\partial h},
$$

with the initial condition corresponding to two planar bubble walls far away from each other and moving in opposite directions (given approximately by $h_{l_{w}}$ in the limit $t \rightarrow-\infty$ ). In the ultrarelativistic limit the ansatz (2.3) will also be an approximate solution of (2.4) before the bubble collision ${ }^{4}$ (for $t<0$ ). In this limit, the kinetic energy per unit area contained in the field configuration $h(z, t)$ prior to the collision is given by

$$
\frac{E_{w}}{A}=\frac{2}{3} v_{T}^{2} \frac{\gamma_{w}}{l_{w}} .
$$

At the moment of the collision, the field configuration makes an "excursion" to field values larger than $v_{T}$ in a small region around the collision point [31] (resulting in $\partial V / \partial h \neq$ 0 in this region). The subsequent evolution of $h(z, t)$ strongly depends on the shape of the potential $V(h)$. The field close to the collision region oscillates back after the initial "kick" in field space, and for a potential with nearly degenerate minima this oscillation is able to drive the field over the potential barrier and into the basin of attraction of the symmetric minimum (figure 1 - Left), where it will perform small-amplitude oscillations. In this

\footnotetext{
${ }^{4}$ Each bubble wall in (2.3) interpolates between the symmetric and broken minima of $V(h)$, and so $\partial V(h) / \partial h=0$ outside the bubble wall. Then, for very thin walls the equation of motion approximately simplifies (before the collision) to $\left(\partial_{t}^{2}-\partial_{z}^{2}\right) h(z, t)=0$, for which any function of the form $f(z+t)$ or $f(z-t)$ is a solution.
} 

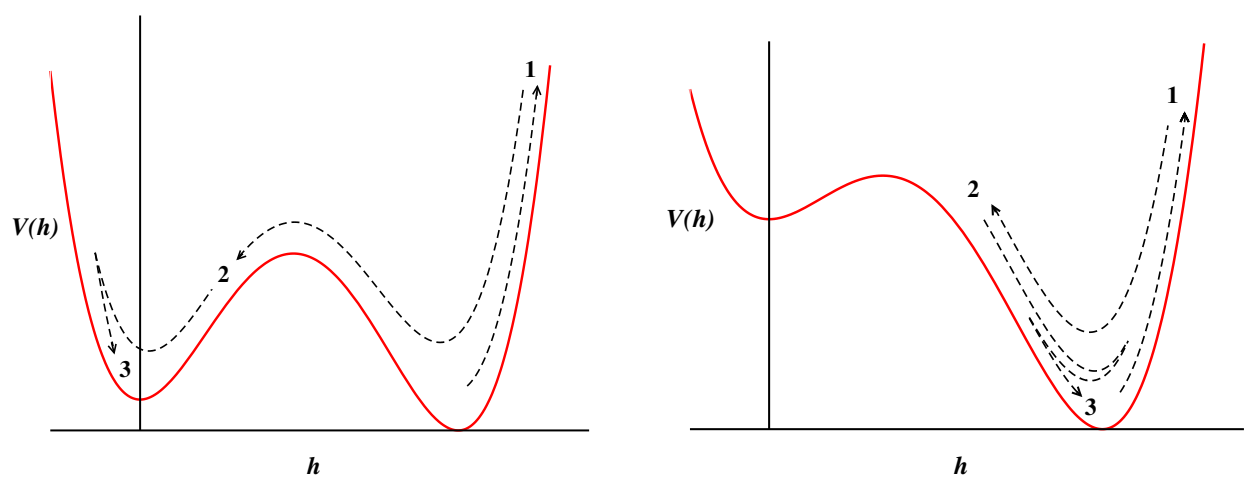

Figure 1. LEFT: potential with nearly degenerate minima. RIGHT: potential with very nondegenerate minima. Each one shows the behaviour of the field immediately after the collision in the region close to the collision point, as described in the text: 1) "Kick" to field values larger than $v(T)$. 2) Large field oscillation, successful (LEFT) or unsuccessful (RIGHT) in driving the field over the potential barrier. 3) Oscillations around the symmetric (LEFT) or broken (RIGHT) minimum.

case the collision is approximately elastic as described above, with the bubble walls being effectively reflected as a region of symmetric phase is re-established between them. The walls move then away from each other until vacuum pressure makes them approach and collide again, repeating the process several times. In each collision some fraction of the energy stored in the walls is radiated into scalar waves and quanta of the fields coupled to $h$, until all of the energy in the walls is radiated away. In contrast to this scenario, for a potential $V(h)$ with very non-degenerate minima (figure 1 - Right), the field oscillation after the "kick" in the region close to the collision point does not effectively drive the field over the potential barrier. As a consequence, the field stays in the basin of attraction of the broken minimum $v_{T}$ and performs relatively large-amplitude oscillations around it, giving rise to a large amount of energy radiated into scalar waves (as opposed to the previous scenario). In this case the collision is very inelastic.

Following [14], we compute the numerical solution for the field profile $h(z, t)$ corresponding to the collision of two bubble walls, obtained from solving (2.4) with a toy potential $V(h)$ of the form

$$
V(h)=a^{2} h^{2}-b^{2} h^{3}+\lambda h^{4}
$$

both in the case of nearly degenerate minima (figure 1 - Left) and very non-degenerate minima (figure 1 - Right). The results are shown in figure 2 (similar plots appeared earlier in [32]). figure 2 - Left (corresponding to the potential of figure 1 - Left) shows an approximately elastic bubble collision, while figure 2 - Right (corresponding to the potential of figure 1 - Right) shows a very inelastic one.

Guided by the numerical solution for $h(z, t)$ in the case of a very inelastic collision, we can obtain an analytic solution $h(z, t)=h_{\mathrm{TI}}$ for the case of a "totally inelastic collision" (as opposed to the "perfectly elastic collision" described earlier), in which all the energy is radiated in the form of scalar waves after the bubble collision. For $t<0$ (before the 

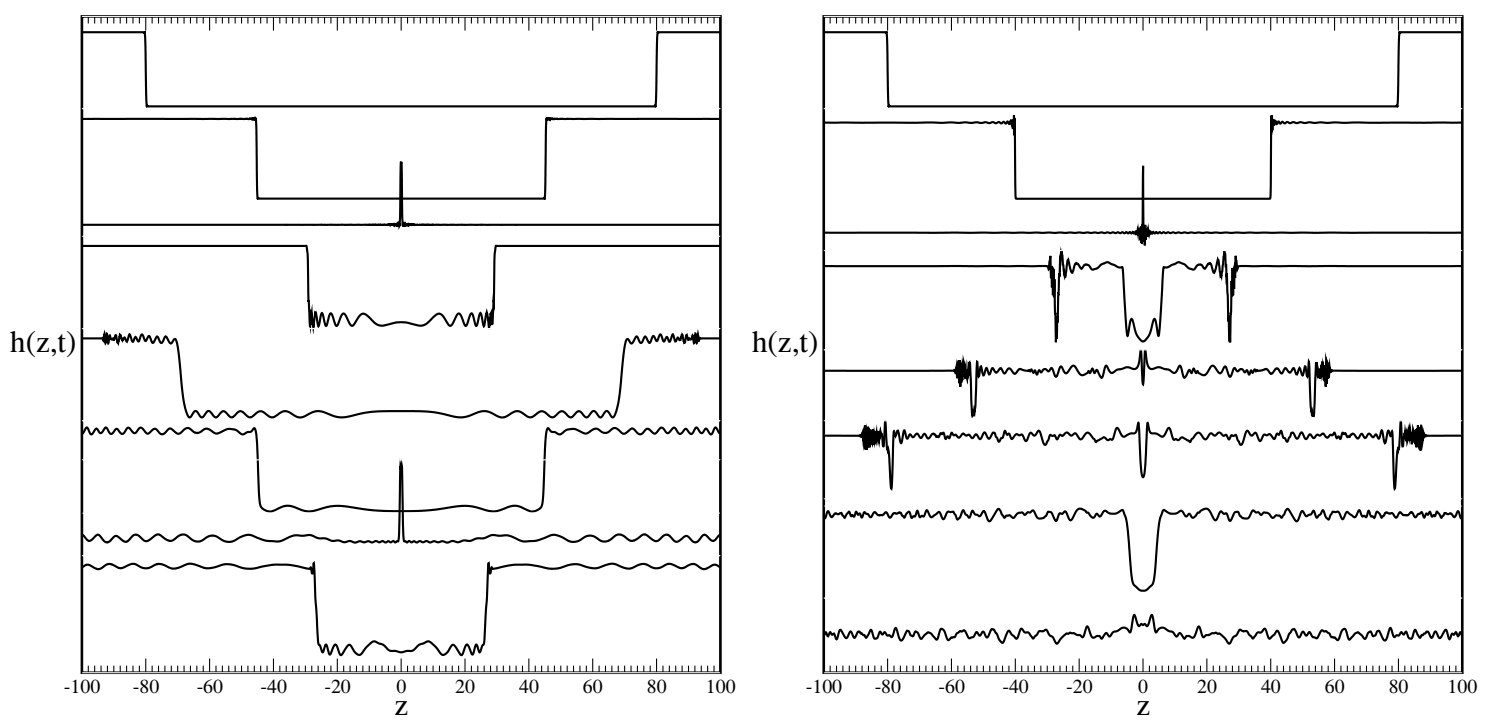

Figure 2. Snapshots of the field profile $h(z, t)$ during a bubble collision ( $t$ increasing downwards). LEFT: bubble collision for the potential with nearly degenerate minima (figure 1 - Left). RIGHT: bubble collision for the potential with very non-degenerate minima (figure 1 - Right). In both cases, $\gamma_{w}=10^{2}, l_{w}=15 / T_{\mathrm{EW}}$ and $T_{\mathrm{EW}}=100 \mathrm{GeV}$.

collision) we have

$$
h_{\mathrm{TI}}(z, t<0)=v_{T}+\frac{v_{T}}{2}\left[\operatorname{Tanh}\left(\gamma_{w} \frac{z+t}{l_{w}}\right)-\operatorname{Tanh}\left(\gamma_{w} \frac{z-t}{l_{w}}\right)\right]
$$

which matches $h_{l_{w}}(z, t<0)$. In order to obtain $h_{\mathrm{TI}}(z, t)$ for $t>0$, we note that the field will not leave the basin of attraction of the broken minimum $v_{T}$ after the collision. We can then approximate the potential $V(h)$ the field will feel for $t>0$ as

$$
V(h) \simeq \frac{m_{h}^{2}}{2}\left(h-v_{T}\right)^{2}=\frac{m_{h}^{2}}{2} \delta h^{2}
$$

This allows to solve the equation of motion (2.4) explicitly for $\delta h_{\mathrm{TI}}(z, t) \equiv h_{\mathrm{TI}}(z, t)-v_{T}$ :

$$
\begin{aligned}
\left(\partial_{t}^{2}-\partial_{z}^{2}\right) \delta h_{\mathrm{TI}}(z, t) & =-m_{h}^{2} \delta h_{\mathrm{TI}}(z, t) \\
\delta h_{\mathrm{TI}}(z, 0) & =h_{l_{w}}(z, 0)-v_{T}=0 \\
\partial_{t} \delta h_{\mathrm{TI}}(z, 0) & =\frac{v_{T} \gamma_{w}}{l_{w}\left[\operatorname{Cosh}\left(\frac{\gamma_{w} z}{l_{w}}\right)\right]^{2}}
\end{aligned}
$$

where the boundary conditions follow from imposing continuity of $\delta h_{\mathrm{TI}}(z, t)$ and $\partial_{t} \delta h_{\mathrm{TI}}(z, t)$ at $t=0$. From (2.9), we finally obtain

$$
h_{\mathrm{TI}}(z, t>0)=v_{T}\left[1+\frac{l_{w}}{\gamma_{w}} \int_{0}^{\infty} d p_{z} \frac{p_{z}}{\sqrt{p_{z}^{2}+m_{h}^{2}}} \frac{\operatorname{Cos}\left(p_{z} z\right)}{\operatorname{Sinh}\left(\frac{\pi l_{w} p_{z}}{2 \gamma_{w}}\right)} \operatorname{Sin}\left(\sqrt{p_{z}^{2}+m_{h}^{2}} t\right)\right]
$$

Notice that in the limit $m_{h} \rightarrow 0,(2.9)$ becomes $\left(\partial_{t}^{2}-\partial_{z}^{2}\right) \delta h_{\mathrm{TI}}(z, t)=0$ and $(2.7)$ is also a solution for $t>0$, case in which the two bubble walls would pass through each other without actually colliding. 
The analysis for the dynamics of bubble collisions presented here may be extended to phase transitions involving multiple fields (see for example [31]), although in this case the analysis of the field evolution after the bubble collision becomes much more complicated (since the scalar potential is multidimensional and the field "excursion" at the moment of the bubble collision will involve several fields), and we will not attempt it here.

\subsection{Particle production through bubble collisions}

The bubble collision processes analyzed in the previous section allow to liberate into the plasma the energy contained in the bubble walls. This can happen either via direct particle production in the collisions or via radiation of classical scalar waves which will subsequently decay into particles. For bubble collisions taking place in a thermal environment, the number densities $n_{\alpha}$ for the different particle species created during the collisions should very quickly approach the ones in thermal equilibrium $n_{\alpha}^{\mathrm{EQ}}$ after the phase transition, thus rendering the particle production process irrelevant for the subsequent evolution of the Universe. However, as it has been briefly discussed in the introduction, under certain conditions fast thermalization of certain species after the phase transition may be avoided, which can make the particle production process very important in that case.

In order to study the particle production through bubble collisions, we will treat the scalar field configuration $h(z, t)$ as a classical external field and the states coupled to it as quantum fields in the presence of this source. In doing so, we will neglect the back-reaction of particle production on the evolution of the bubble walls themselves throughout the collision, which should be a good approximation when the energy of the produced particles (for each species) is much less than the energy contained in the field configuration $h(z, t)$. The probability of particle production is given by [13]

$$
\mathcal{P}=2 \operatorname{Im}(\Gamma[h]) \quad(\mathcal{P} \ll 1)
$$

where $\Gamma[h]$ is the effective action. $\Gamma[h]$ is the generating functional of 1 PI Green functions, and to the quadratic order in $h$

$$
\Gamma[h]=\frac{1}{2} \int d^{4} x_{1} d^{4} x_{2} h\left(x_{1}\right) h\left(x_{2}\right) \Gamma^{(2)}\left(x_{1}, x_{2}\right)
$$

with $\Gamma^{(2)}\left(x_{1}, x_{2}\right) \equiv \Gamma^{(2)}\left(x_{1}-x_{2}\right)$ being the 2-point 1PI Green function. In terms of its Fourier transform $\tilde{\Gamma}^{(2)}\left(p^{2}\right)$, and using (2.11) and (2.12) we get

$$
\mathcal{P}=\int \frac{d^{4} p}{(2 \pi)^{4}} \operatorname{Im}\left(\tilde{\Gamma}^{(2)}\left(p^{2}\right)\right) \int d^{4} x_{1} d^{4} x_{2} h\left(x_{1}\right) h\left(x_{2}\right) e^{i p\left(x_{1}-x_{2}\right)}
$$

The last integral in $(2.13)$ is just $|\tilde{h}(p)|^{2}$, with $\tilde{h}(p)$ being the Fourier transform of the Higgs field configuration $h(x)$

$$
\tilde{h}(p)=\int d^{4} x h(x) e^{i p x}
$$


For a background field configuration $h(z, t)$, its Fourier transform is given by $\tilde{h}(p)=$ $(2 \pi)^{2} \delta\left(p_{x}\right) \delta\left(p_{y}\right) \tilde{h}\left(p_{z}, \omega\right)$. Then, using (2.13), we obtain the mean number of particles produced per unit area [13]:

$$
\frac{\mathcal{N}}{A}=2 \int \frac{d p_{z} d \omega}{(2 \pi)^{2}}\left|\tilde{h}\left(p_{z}, \omega\right)\right|^{2} \operatorname{Im}\left(\tilde{\Gamma}^{(2)}\left(\omega^{2}-p_{z}^{2}\right)\right)
$$

The physical interpretation of (2.15) is rather simple [13]: the scalar field configuration $h(z, t)$, corresponding to the two bubble walls that approach and collide, can be decomposed into modes of definite four-momentum $p^{2}=\omega^{2}-p_{z}^{2}$ via the Fourier transform. Modes with $p^{2}>0$ represent propagating field quanta with mass squared $m^{2}=p^{2}$. Then, $(2.15)$ integrates over the amount of field quanta of mass $p^{2}$ contained in the field configuration multiplied by the probability of those quanta to decay.

The Fourier transform of the background field configuration $h(z, t)$ can be performed explicitly both for the case of a perfectly elastic collision and of a totally inelastic one analyzed in the previous section. For a perfectly elastic collision, in the limit of infinitely thin walls $\left(h(z, t)=h_{\infty}\right)$, we obtain

$$
\tilde{h}\left(p_{z}, \omega\right)=\tilde{h}_{\infty}\left(p_{z}, \omega\right) \equiv \frac{4 v_{T}}{\omega^{2}-p_{z}^{2}}
$$

However, since the highest values of $p_{z}$ and $\omega$ available in the field configuration are naively expected to be of order $\gamma_{w} / l_{w}$ (modes with $p_{z}, \omega \gg \gamma_{w} / l_{w}$ will be exponentially damped), the integration in (2.15) should in this case be cut-off for $p_{z}>\gamma_{w} / l_{w}$ and $\omega>\gamma_{w} / l_{w}$. From $(2.15)$ and $(2.16)$ we then obtain

$$
\frac{\mathcal{N}_{\infty}}{A}=\frac{32 v_{T}^{2}}{\pi^{2}} \int_{0}^{\frac{\gamma w}{l_{w}}} d \omega \int_{0}^{\frac{\gamma w}{l_{w}}} d p_{z} \frac{\operatorname{Im}\left(\tilde{\Gamma}^{(2)}\left(\omega^{2}-p_{z}^{2}\right)\right)}{\left(\omega^{2}-p_{z}^{2}\right)^{2}}
$$

Alternatively, when the thickness of the bubble walls is accounted for $\left(h(z, t)=h_{l_{w}}\right)$, the Fourier transform of (2.3) gives

$$
\tilde{h}\left(p_{z}, \omega\right)=\tilde{h}_{l_{w}}\left(p_{z}, \omega\right) \equiv \frac{\pi l_{w} \omega}{2 \gamma_{w}} \frac{4 v_{T}}{\operatorname{Sinh}\left[\frac{\pi l_{w} \omega}{2 \gamma_{w}}\right]} \frac{1}{\omega^{2}-p_{z}^{2}}
$$

which automatically incorporates the exponential damping for $\omega, p_{z} \gg \gamma_{w} / l_{w}$. The mean number of particles per unit area now reads

$$
\frac{\mathcal{N}_{l_{w}}}{A}=\frac{8 v_{T}^{2} l_{w}^{2}}{\gamma_{w}^{2}} \int_{0}^{\infty} d \omega \int_{0}^{\infty} d p_{z} \frac{\operatorname{Im}\left(\tilde{\Gamma}^{(2)}\left(\omega^{2}-p_{z}^{2}\right)\right)}{\left(\omega^{2}-p_{z}^{2}\right)^{2}} \frac{\omega^{2}}{\left(\operatorname{Sinh}\left[\frac{\pi l_{w} \omega}{2 \gamma_{w}}\right]\right)^{2}}
$$

For the opposite case of a totally inelastic collision $\left(h(z, t)=h_{\mathrm{TI}}\right)$, the Fourier transform is given by

$$
\tilde{h}\left(p_{z}, \omega\right)=\tilde{h}_{\mathrm{TI}}\left(p_{z}, \omega\right) \equiv \frac{\pi l_{w} p_{z}}{2 \gamma_{w}} \frac{2 v_{T}}{\operatorname{Sinh}\left[\frac{\pi l_{w} p_{z}}{2 \gamma_{w}}\right]}\left(\frac{1}{\omega^{2}-p_{z}^{2}}-\frac{1}{\omega^{2}-p_{z}^{2}-m_{h}^{2}}\right)
$$


The relative "-" sign between the two contributions in (2.20) can be easily understood noticing that in the limit $m_{h} \rightarrow 0$ the Fourier transform of $h_{\mathrm{TI}}(z, t)$ should give $\tilde{h}\left(p_{z}, \omega\right) \sim$ $\delta\left(\omega \pm p_{z}\right)$. From (2.20), the mean number of particles produced per unit area in the case of a totally inelastic collision is given by

$$
\frac{\mathcal{N}_{\mathrm{TI}}}{A}=\frac{2 v_{T}^{2} l_{w}^{2}}{\gamma_{w}^{2}} \int_{0}^{\infty} d \omega \int_{0}^{\infty} d p_{z} \frac{m_{h}^{4} \operatorname{Im}\left(\tilde{\Gamma}^{(2)}\left(\omega^{2}-p_{z}^{2}\right)\right)}{\left(\omega^{2}-p_{z}^{2}\right)^{2}\left(\omega^{2}-p_{z}^{2}-m_{h}^{2}\right)^{2}} \frac{p_{z}^{2}}{\left(\operatorname{Sinh}\left[\frac{\pi l_{w} p_{z}}{2 \gamma_{w}}\right]\right)^{2}}
$$

The expressions $(2.17),(2.19)$ and $(2.21)$ can be rewritten in a more compact form by making the change of variables $\chi=\omega^{2}-p_{z}^{2}, \Psi=\omega^{2}+p_{z}^{2}$. After performing the integral in $\Psi$, the mean number of particles produced per unit area finally reads

$$
\frac{\mathcal{N}}{A}=\frac{1}{2 \pi^{2}} \int_{0}^{\infty} d \chi f(\chi) \operatorname{Im}\left(\tilde{\Gamma}^{(2)}(\chi)\right)
$$

The function $f(\chi)$ encodes the details of the bubble collision process and quantifies the efficiency of particle production. For a perfectly elastic collision, in the limit of infinitely thin bubble walls, we have

$$
f(\chi)=f_{\infty}(\chi) \equiv \frac{16 v_{T}^{2} \log \left[\frac{2\left(\frac{\gamma_{w}}{l_{w}}\right)^{2}-\chi+2 \frac{\gamma_{w}}{l_{w}} \sqrt{\left(\frac{\gamma_{w}}{l_{w}}\right)^{2}-\chi}}{\chi}\right]}{\chi^{2}} \Theta\left[\left(\frac{\gamma_{w}}{l_{w}}\right)^{2}-\chi\right]
$$

For a perfectly elastic collision, and for bubble walls with finite thickness, we have

$$
f(\chi)=f_{l_{w}}(\chi) \equiv \frac{2 \pi^{2} l_{w}^{2} v_{T}^{2}}{\gamma_{w}^{2}} \frac{1}{\chi^{2}} \int_{\chi}^{\infty} d \Psi \frac{\Psi+\chi}{\sqrt{\Psi^{2}-\chi^{2}}} \frac{1}{\left(\operatorname{Sinh}\left[\frac{\pi l_{w} \sqrt{\Psi+\chi}}{2 \sqrt{2} \gamma_{w}}\right]\right)^{2}}
$$

Finally, for a totally inelastic collision, we have

$$
f(\chi)=f_{\mathrm{TI}}(\chi) \equiv \frac{\pi^{2} l_{w}^{2} v_{T}^{2}}{2 \gamma_{w}^{2}} \frac{m_{h}^{4}}{\chi^{2}\left(\chi-m_{h}^{2}\right)^{2}} \int_{\chi}^{\infty} d \Psi \frac{\Psi-\chi}{\sqrt{\Psi^{2}-\chi^{2}}} \frac{1}{\left(\operatorname{Sinh}\left[\frac{\pi l_{w} \sqrt{\Psi-\chi}}{2 \sqrt{2} \gamma_{w}}\right]\right)^{2}}
$$

In figure 3 we compare the efficiency $f(\chi)$ for the various cases (2.23), (2.24) and (2.25). Notice that $f_{\text {TI }}(\chi)$ diverges as $\chi \rightarrow m_{h}^{2}$. This divergence is artificial, due to considering $h(z, t)$ over infinite time and space, and should be cut-off since our solution is not valid over distances larger than the bubble radius $R_{B}$. Implementing this cut-off can be well approximated by replacing in $(2.24)$

$$
\left(\chi-m_{h}^{2}\right)^{2} \rightarrow\left(\chi-m_{h}^{2}\right)^{2}+\left(m_{h}^{6} l_{w}^{2}\right) / \gamma_{w}^{2}
$$

Defining $\chi_{\min }$ as the minimum value of $\chi$ for which particle production is possible (corresponding to the squared sum of the masses $M_{\alpha}$ of the particles being produced), we immediately see from figure 3 that for a totally inelastic collision, production of light particles $\left(\chi_{\min }<m_{h}^{2}\right)$ may be very efficient, while production of heavy particles $\left(\chi_{\min } \gg\right.$ 

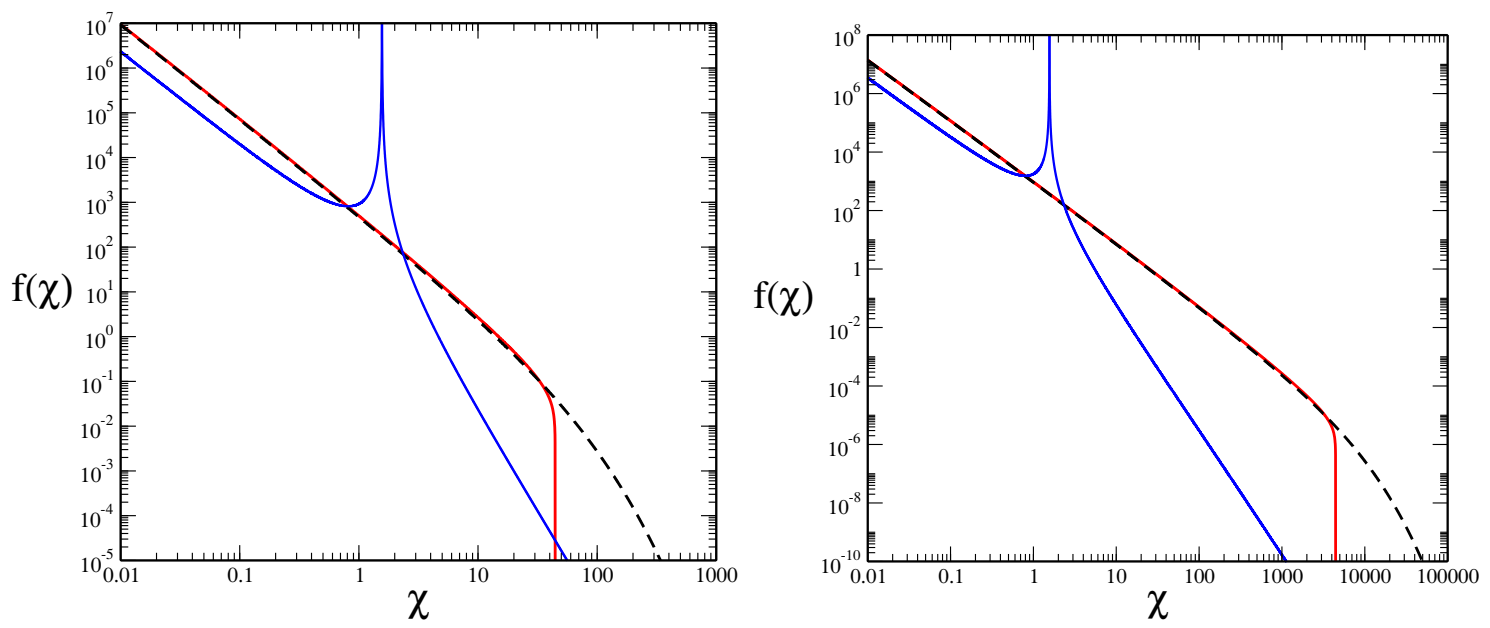

Figure 3. Particle production efficiency $f\left(\chi \equiv \omega^{2}-p_{z}^{2}\right)$ for $\gamma_{w}=10^{2}$ (LEFT) and $\gamma_{w}=10^{3}$ (RIGHT), $l_{w}=15 / T_{\mathrm{EW}}$ and $T_{\mathrm{EW}}=100 \mathrm{GeV}$, in the case of a perfectly elastic collision with infinitely thin bubble walls (2.23) (solid red) and with a finite bubble wall thickness (2.24) (dashedblack), and in the case of a totally inelastic collision (2.25) (solid blue) with $m_{h}=125 \mathrm{GeV}$. The $\chi$-axis is displayed in units of $(100 \mathrm{GeV})^{2}$.

$m_{h}^{2}$ ) will be extremely suppressed. For a perfectly elastic collision, however, the production of heavy particles may be relatively efficient (we will comment further on this point at the end of section 3). For the study of the efficiency of particle production in varios different scenarios in the next sections, we will use (2.23) for the case of an elastic collision, while for the case of a very inelastic one it is possible to show that (2.25) (together with (2.26)) can be approximated as

$$
f_{\mathrm{TI}}(\chi) \simeq 4 v_{T}^{2} m_{h}^{4} \frac{\log \left[\frac{2\left(\frac{\gamma_{w}}{l_{w}}\right)^{2}+\chi+2 \frac{\gamma_{w}}{l_{w}} \sqrt{\left(\frac{\gamma_{w}}{l_{w}}\right)^{2}+\chi}}{\chi}\right]}{\chi^{2}\left[\left(\chi-m_{h}^{2}\right)^{2}+m_{h}^{6} \frac{l_{w}^{2}}{\gamma_{w}^{2}}\right]} .
$$

Let us now turn to the evaluation of the imaginary part of the 2-point 1PI Green function's Fourier transform $\tilde{\Gamma}^{(2)}\left(\chi \equiv \omega^{2}-p_{z}^{2}\right)$. Through the optical theorem, we can write:

$$
\operatorname{Im}\left(\tilde{\Gamma}^{(2)}(\chi)\right)=\frac{1}{2} \sum_{\alpha} \int d \Pi_{\alpha}|\overline{\mathcal{M}}(h \rightarrow \alpha)|^{2} \Theta\left[\chi-\chi_{\min }\right]
$$

where $|\overline{\mathcal{M}}(h \rightarrow \alpha)|^{2}$ is the spin-averaged squared amplitude for the decay of $h$ into a set of particles $\alpha$ with masses $M_{\alpha}, \chi_{\min } \equiv\left(\sum M_{\alpha}\right)^{2}$ is the minimum value of $\chi$ for which this decay is possible and $d \Pi_{\alpha}$ is the relativistically invariant $n$-body phase space element

$$
d \Pi_{\alpha}=\left(\prod_{i} \frac{d^{3} k_{i}}{(2 \pi)^{3}} \frac{1}{2 E_{i}}\right)(2 \pi)^{4} \delta^{4}\left(p-\sum_{i} k_{i}\right)
$$


Then, the number of particles of a certain type $\alpha$ produced per unit area during the bubble collision will simply read from (2.22) and (2.28)

$$
\left.\frac{\mathcal{N}}{A}\right|_{\alpha}=\frac{1}{4 \pi^{2}} \int_{\chi_{\min }}^{\infty} d \chi f(\chi) \int d \Pi_{\alpha}|\overline{\mathcal{M}}(h \rightarrow \alpha)|^{2}
$$

The amount of energy produced per unit area in the form of particles $\alpha$ is obtained by weighting (2.30) by the energy of each decaying Fourier mode. This yields

$$
\left.\frac{\mathcal{E}}{A}\right|_{\alpha}=\frac{1}{4 \pi^{2}} \int_{\chi_{\min }}^{\infty} d \chi f(\chi) \sqrt{\chi} \int d \Pi_{\alpha}|\overline{\mathcal{M}}(h \rightarrow \alpha)|^{2}
$$

From (2.30) and (2.31), the non-thermally produced energy density $\rho_{\alpha}$ (assuming that the produced particles quickly diffuse into the bubble interior) reads

$$
\left.\rho_{\alpha} \equiv \frac{\mathcal{E}}{V}\right|_{\alpha}=\left.\left.\frac{\mathcal{E}}{A}\right|_{\alpha} \frac{A}{V} \simeq \frac{\mathcal{E}}{A}\right|_{\alpha} \frac{3}{2 R_{B}}
$$

with $A \sim 4 \pi R_{B}^{2}$ being the total collision area and $V$ the volume of the two colliding bubbles. From (2.32), and bearing in mind that $R_{B} \simeq \beta^{-1}$, the non-thermally generated comoving energy density is

$$
\Upsilon_{\alpha}=\left.\frac{\rho_{\alpha}}{s\left(T_{\mathrm{EW}}\right)} \simeq \frac{20}{\sqrt{\pi g_{*}}} \frac{1}{M_{\mathrm{Pl}} T_{\mathrm{EW}}} \frac{\beta}{H} \frac{\mathcal{E}}{A}\right|_{\alpha}
$$

with $s\left(T_{\mathrm{EW}}\right)$ the entropy density after the EW phase transition.

\section{Particle production via the Higgs portal}

The efficiency of particle production may strongly depend on the nature of the particles being produced. In this section we will analyze the particle production efficiency for scalars $S$, fermions $f$ and vector bosons $V_{\mu}$ coupled to the Higgs field. Apart from estimating the production of SM fermions and gauge bosons through this process, we will consider a simple Higgs-portal extension of the SM in order to study the production of other possible scalar, fermion or vector boson particles. Furthermore, we will restrict ourselves to $Z_{2}$ symmetric Higgs-portal scenarios, since we will ultimately be interested in dark matter analyses. We also comment on how to interpret the results in the case when the calculated particle production exceeds the energy available in the bubble wall.

\subsection{Scalars}

For the complex scalar $S$ interacting with the SM via the Higgs portal, the relevant part of the lagrangian is given by

$$
-\Delta \mathcal{L}_{s}=m_{s}^{2}|S|^{2}+\lambda_{s}|H|^{2}|S|^{2} \quad \text { with } \quad H=\left(\begin{array}{c}
0 \\
\frac{h+v_{T}}{\sqrt{2}}
\end{array}\right) .
$$



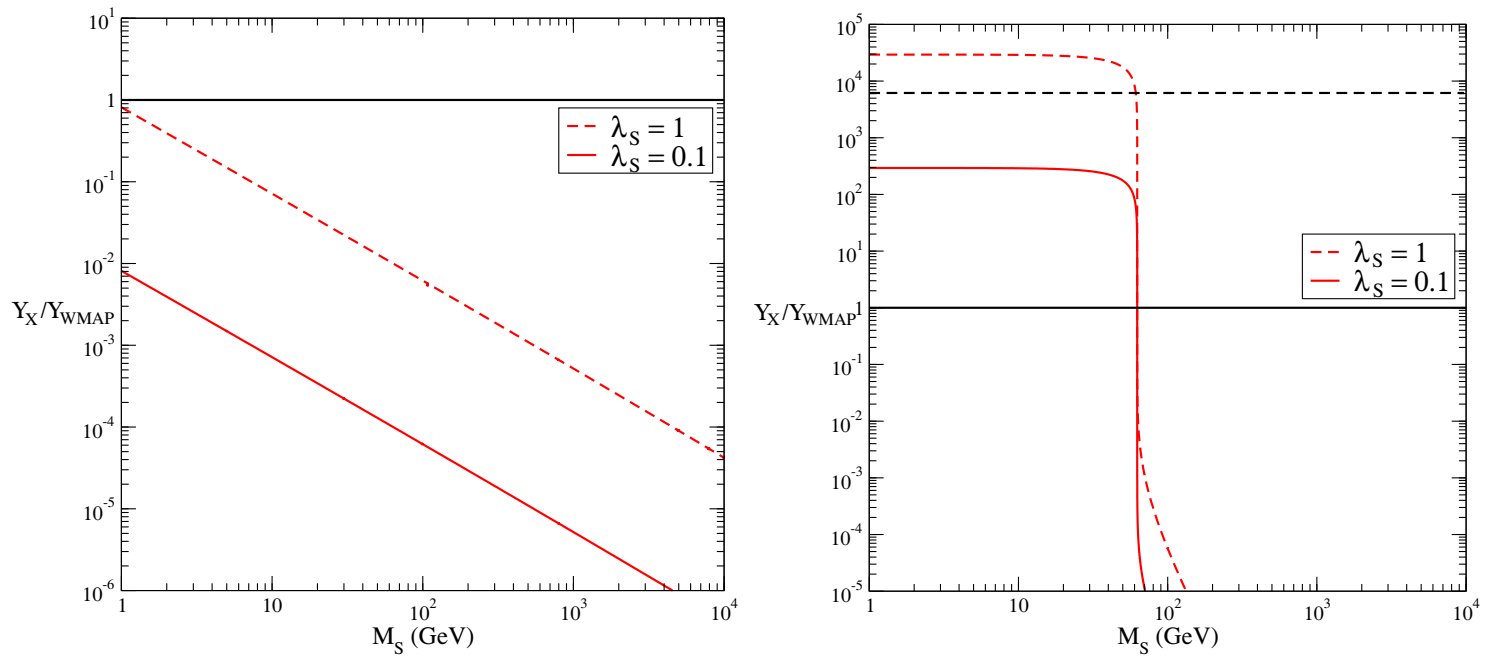

Figure 4. Generated comoving energy density $\Upsilon$ in scalars $S$ (normalized to the observed dark matter comoving energy density) as a function of the scalar mass $M_{s}$ in the perfectly elastic collision limit (LEFT) and totally inelastic collision limit (RIGHT) for $\gamma_{w}=10^{8}, l_{w}=15 / T_{\mathrm{EW}}$ and $T_{\mathrm{EW}}=$ $100 \mathrm{GeV}$. The solid-black line corresponds to the observed dark matter comoving energy density, and the dashed-black line (not seen in LEFT) corresponds to the maximum possibly generated comoving energy density $\left(\mathcal{E}=E_{w}\right)$.

In this case, $|\mathcal{M}(h \rightarrow S \bar{S})|^{2}=\lambda_{s}^{2} v_{T}^{2}$, and one immediately obtains

$$
\operatorname{Im}\left[\tilde{\Gamma}^{(2)}(\chi)\right]_{S}=\lambda_{s}^{2} v_{T}^{2} \int d \Pi_{S}=\sqrt{1-4 \frac{M_{s}^{2}}{\chi}} \frac{\lambda_{s}^{2} v_{T}^{2}}{8 \pi} \Theta\left(\chi-4 M_{s}^{2}\right)
$$

with $M_{s}^{2} \equiv m_{s}^{2}+\left(\lambda_{s} / 2\right) v_{T}^{2}$ being the scalar squared mass. Then, using (2.22), (2.23), (2.27), (2.33) and (3.2) we can compute the $S$-scalar comoving energy density generated through the bubble collisions (normalized to the observed dark matter comoving energy density) as a function of $M_{s}$ and $\lambda_{s}$. The results are shown in figure 4 .

From figure 4 it can be clearly seen that scalar particle production is quite suppressed for elastic collisions. For very inelastic collisions, heavy-scalar particle production is extremely suppressed, while production of light scalars turns out to be very efficient in this case. In fact, figure 4 shows that for large values of $\lambda_{s}\left(\lambda_{s} \lesssim 1\right)$ the naively calculated energy of the produced particles $\mathcal{E}$ exceeds the amount of energy on the bubble walls $E_{w}$. That inconsistency indicates that in these cases backreaction cannot be neglected. We will comment and expand on this issue in section 3.4.

\subsection{Fermions}

Turning now to fermionic particle production, in the presence of a tree-level Yukawa coupling between the Higgs and the fermions $\lambda_{f} H \bar{f} f$, the squared decay amplitude reads

$$
|\overline{\mathcal{M}}(h \rightarrow \bar{f} f)|^{2}=2 \lambda_{f}^{2}\left(p^{2}-4 m_{f}^{2}\right)
$$


which, in the case of SM fermions, leads directly to

$$
\operatorname{Im}\left[\tilde{\Gamma}^{(2)}(\chi)\right]_{f}=\frac{m_{f}^{2}}{4 \pi v_{T}^{2}} \chi\left(1-\frac{4 m_{f}^{2}}{\chi}\right)^{\frac{3}{2}} \Theta\left(\chi-4 m_{f}^{2}\right)
$$

The production of (SM) fermions will then be enhanced with respect to the one of Higgs-portal $S$-scalars (specially in the limit of very elastic collisions, see figure 5 ) due to the extra factor $\left(\chi-4 m_{f}^{2}\right)$ in (3.4). Scenarios where the fermionic particle production might be important include (apart from the SM itself) the MSSM and its various extensions, due to the tree-level coupling between Higgses, Higgsinos and Gauginos. ${ }^{5}$

In the absence of a direct coupling, the interaction between the Higgs and the fermions will occur via an effective operator. This is the case for the so-called fermionic Higgs-portal:

$$
-\Delta \mathcal{L}_{f}=m_{f} \bar{f} f+\frac{\lambda_{f}}{\Lambda}|H|^{2} \bar{f} f
$$

However, since bubble collisions may excite very massive Higgs field modes $\left(p^{2} \gg\right.$ $T_{\mathrm{EW}}^{2}$ ), particle production in this case may be sensitive to the UV completion of the Higgsportal effective theory, making it unreliable to compute the particle production in the fermionic Higgs-portal via (3.5). Here we consider a simple UV completion for the fermionic Higgs-portal, and compute the particle production in this case. We add a singlet scalar field $S$ as a mediator between the Higgs field and the fermion $f$, the relevant part of the lagrangian being

$$
-\Delta \mathcal{L}_{f}=\frac{m_{s}^{2}}{2} S^{2}+\frac{\lambda_{s}}{2}|H|^{2} S^{2}+\mu_{s}|H|^{2} S+m_{f} \bar{f} f+\lambda_{f} S \bar{f} f
$$

For simplicity, we will avoid a vev for $S$ (it can be done through the addition of a linear term for $S$ in (3.6)). For $\mu_{s} \neq 0$ the effective fermionic Higgs-portal operator $|H|^{2} \bar{f} f$ will be generated at tree-level. The squared decay amplitude for $h \rightarrow \bar{f} f$ will then be

$$
|\overline{\mathcal{M}}(h \rightarrow \bar{f} f)|^{2}=2 \frac{\lambda_{f}^{2} \mu_{s}^{2} v_{T}^{2}}{\left(p^{2}-M_{s}^{2}\right)^{2}+\Gamma_{s}^{2} M_{s}^{2}}\left(p^{2}-4 m_{f}^{2}\right)
$$

with

$$
\Gamma_{s}=\frac{\lambda_{s}^{2} v_{T}^{2}+\mu_{s}^{2}}{16 \pi M_{s}} \sqrt{1-\frac{4 m_{h}^{2}}{M_{s}^{2}}} \Theta\left(M_{s}^{2}-4 m_{h}^{2}\right)+\frac{\lambda_{f}^{2} M_{s}}{8 \pi}\left(1-\frac{4 m_{f}^{2}}{M_{s}^{2}}\right)^{\frac{3}{2}} \Theta\left(M_{s}^{2}-4 m_{f}^{2}\right)
$$

leading finally to

$$
\operatorname{Im}\left[\tilde{\Gamma}^{(2)}(\chi)\right]_{f}=\frac{\lambda_{f}^{2} \mu_{s}^{2} v_{T}^{2}}{4 \pi} \frac{\chi}{\left(\chi-M_{s}^{2}\right)^{2}+\Gamma_{s}^{2} M_{s}^{2}}\left(1-\frac{4 m_{f}^{2}}{\chi}\right)^{\frac{3}{2}} \Theta\left(\chi-4 m_{f}^{2}\right)
$$

\footnotetext{
${ }^{5}$ In particular, the production of neutralino dark matter might have an impact on the subsequent evolution of the Universe.
} 

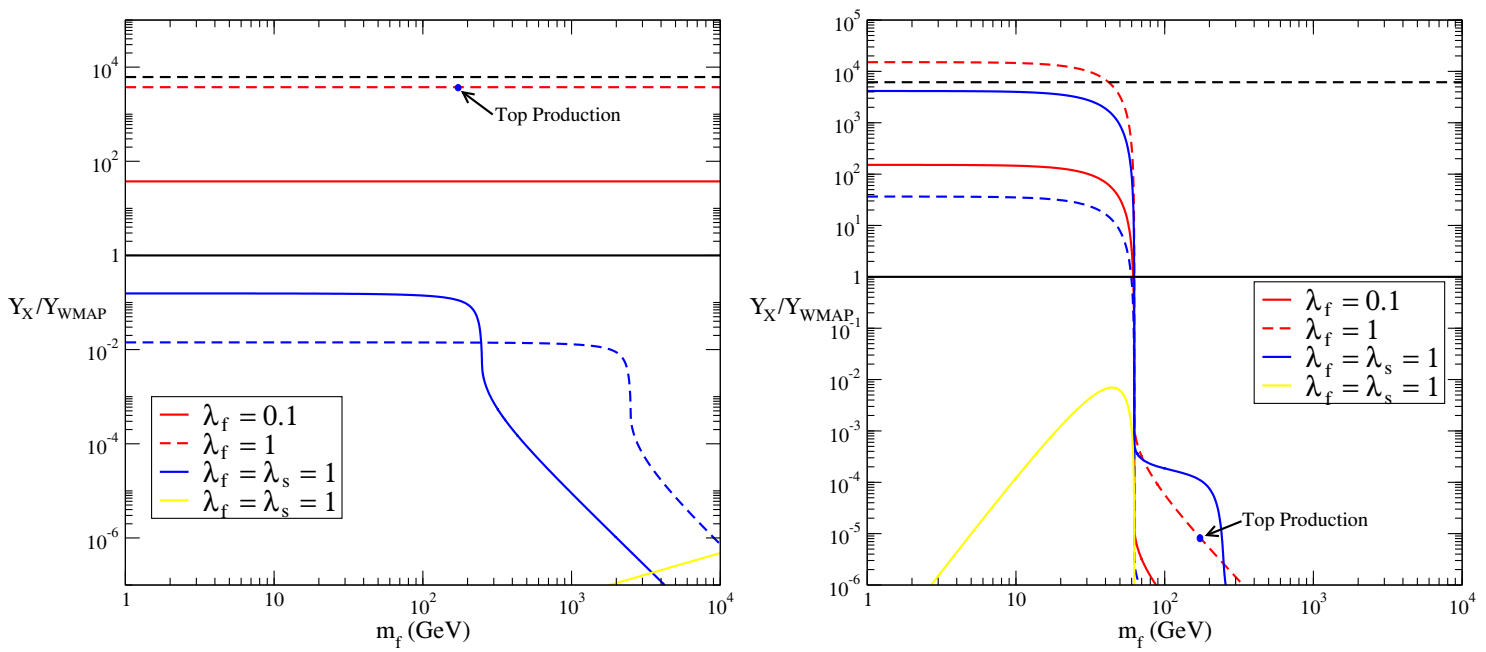

Figure 5. Generated comoving energy density $\Upsilon$ in fermions $f$ (normalized to the observed dark matter comoving energy density) as a function of the fermion mass $m_{f}$ in the perfectly elastic collision limit (LEFT) and totally inelastic collision limit (RIGHT) for $\gamma_{w}=10^{8}, l_{w}=15 / T_{\mathrm{EW}}$ and $T_{\mathrm{EW}}=100 \mathrm{GeV}$. Red lines: production in the presence of a direct tree-level Yukawa coupling between fermions and Higgs (3.4). Blue lines: production for a tree-level effective coupling (3.9), for $\mu_{s}=M_{s}=500 \mathrm{GeV}$ (solid) and $5 \mathrm{TeV}$ (dashed). Yellow lines: production for a 1-loop effective coupling (3.10). The solid-black line corresponds to the observed dark matter comoving energy density, and the dashed-black line corresponds to the maximum possible generated comoving number density $\left(\mathcal{E}=E_{w}\right)$.

When $\mu_{s}=0$ the effective fermionic Higgs-portal operator is not generated at treelevel, but rather the decay $h \rightarrow \bar{f} f$ occurs via a finite 1-loop diagram, yielding

$$
\operatorname{Im}\left[\tilde{\Gamma}^{(2)}(\chi)\right]_{f}=\frac{\left(\lambda_{s} \lambda_{f}^{2}\right)^{2}}{(4 \pi)^{5}} F\left[m_{f}^{2}, M_{s}^{2}, \chi\right] \chi\left(1-\frac{4 m_{f}^{2}}{\chi}\right)^{\frac{3}{2}} \Theta\left(\chi-4 m_{f}^{2}\right)
$$

where $F\left[m_{f}^{2}, M_{s}^{2}, \chi\right]$ is a form factor that scales as

$$
F\left[m_{f}^{2}, M_{s}^{2}, \chi\right] \longrightarrow \frac{m_{f}^{4}}{\chi^{2}} \log \left(\frac{\chi}{m_{f}^{2}}\right) \quad \chi \gg m_{f}^{2}, M_{s}^{2}
$$

Fermionic Higgs-portal particle production both in the $\mu_{s}=0$ and $\mu_{s} \neq 0$ is shown in figure 5, where it can be clearly seen that the production in the absence of a direct coupling between the Higgs and the fermions $f$ differs from what would have been naively obtained using (3.5). As for the case of scalar particle production, under certain circumstances the estimate of fermionic particle production neglecting backreaction exceeds the amount of energy stored in the bubble walls $\left(\mathcal{E}>E_{w}\right)$, and in order to obtain a physically meaningful result backreaction should be included (We will expand on this issue in section 3.4).

\subsection{Vector bosons}

Finally, we study the production of vector boson particles. In the presence of a treelevel coupling between the Higgs and the vector bosons $\lambda_{V} M_{V} h V_{\mu} V_{\mu}$, the squared decay 
amplitude reads

$$
\left|\overline{\mathcal{M}}\left(h \rightarrow V_{\mu} V_{\mu}\right)\right|^{2}=\lambda_{V}^{2} M_{V}^{2}\left(3-\frac{p^{2}}{M_{V}^{2}}+\frac{p^{4}}{4 M_{V}^{4}}\right)
$$

leading to

$$
\operatorname{Im}\left[\tilde{\Gamma}^{(2)}(\chi)\right]_{V}=\frac{\lambda_{V}^{2} M_{V}^{2}}{8 \pi}\left(3-\frac{\chi}{M_{V}^{2}}+\frac{\chi^{2}}{4 M_{V}^{4}}\right) \sqrt{1-4 \frac{M_{V}^{2}}{\chi}} \Theta\left(\chi-4 M_{V}^{2}\right)
$$

Comparing (3.2), (3.4) and (3.13) we immediately observe the relative efficiency of particle production for scalars, fermions and vector bosons. While $\operatorname{Im}\left[\tilde{\Gamma}^{(2)}(\chi)\right]$ scales as $\chi^{0}$ for scalars, and as $\chi$ for fermions, in the case of vector bosons it scales as $\chi^{2}$, thus greatly enhancing production of vector bosons with respect to scalars or fermions for very elastic collisions (see figure 6). It is then expected that most of the available energy from the EW phase transition will go into $W_{\mu}$ and $Z_{\mu}$ gauge boson production and (possibly) other vector bosons coupled at tree-level to the Higgs in extensions of the SM. ${ }^{6}$

In the absence of a direct coupling, the interaction between the Higgs and the vector bosons may occur via an effective operator, as in the so-called vector Higgs-portal [34]:

$$
-\Delta \mathcal{L}_{V}=\frac{1}{2} m_{V}^{2} V_{\mu} V^{\mu}+\lambda_{V}|H|^{2} V_{\mu} V^{\mu}
$$

However (like for the fermionic Higgs-portal) an analysis of vector boson particle production in the context of the effective theory (3.14) will be unreliable due to very massive Higgs field modes $\left(p^{2} \gg T_{\mathrm{EW}}^{2}\right)$ being excited during the bubble collisions. Vector boson particle production will then be sensitive to the way in which the effective operator $|H|^{2} V_{\mu} V^{\mu}$ is generated. One possible way of generating the effective operator at tree-level, being $V_{\mu}$ a hidden $\mathrm{U}(1)$ gauge field, is by integrating out a U(1)-charged complex scalar $S$ which has a Higgs portal coupling $|H|^{2} S^{*} S$, the relevant part of the lagrangian then being

$$
-\Delta \mathcal{L}_{V}=\frac{1}{4} F_{\mu \nu} F^{\mu \nu}-D_{\mu} S^{*} D^{\mu} S+V(S)+\lambda_{h s}|H|^{2} S^{*} S
$$

In this scenario, the vector boson $V_{\mu}$ acquires a mass via the spontaneous breaking of the hidden $\mathrm{U}(1)$, through a vev $v_{S}$ for the $S$-scalar. ${ }^{7}$ The squared decay amplitude for $h \rightarrow V_{\mu} V_{\mu}$ will then be

$$
\left|\overline{\mathcal{M}}\left(h \rightarrow V_{\mu} V_{\mu}\right)\right|^{2}=\frac{\lambda_{h s}^{2}}{4} \frac{v_{T}^{2} M_{V}^{4}}{\left(p^{2}-M_{s}^{2}\right)^{2}+\Gamma_{s}^{2} M_{s}^{2}}\left(3-\frac{p^{2}}{M_{V}^{2}}+\frac{p^{4}}{4 M_{V}^{4}}\right)
$$

with $\Gamma_{s}$ being the decay width of $S$. This leads to

$$
\operatorname{Im}\left[\tilde{\Gamma}^{(2)}(\chi)\right]_{V}=\frac{\lambda_{h s}^{2}}{32 \pi} \frac{v_{T}^{2} M_{V}^{4}\left(3-\frac{\chi}{M_{V}^{2}}+\frac{\chi^{2}}{4 M_{V}^{4}}\right)}{\left(\chi-M_{s}^{2}\right)^{2}+\Gamma_{s}^{2} M_{s}^{2}} \sqrt{1-\frac{4 M_{V}^{2}}{\chi}} \Theta\left(\chi-4 M_{V}^{2}\right)
$$

\footnotetext{
${ }^{6}$ Such as Little Higgs theories or extra-dimensional scenarios with gauge fields living in the bulk.

${ }^{7}$ This implies that there may have been another phase transition in the early Universe associated with the spontaneous breaking of the hidden U(1) gauge symmetry, which we need to require to have happened long before the EW phase transition since otherwise the EW phase transition would have been effectively multi-field and our present analysis of particle production would be totally unrealistic.
} 

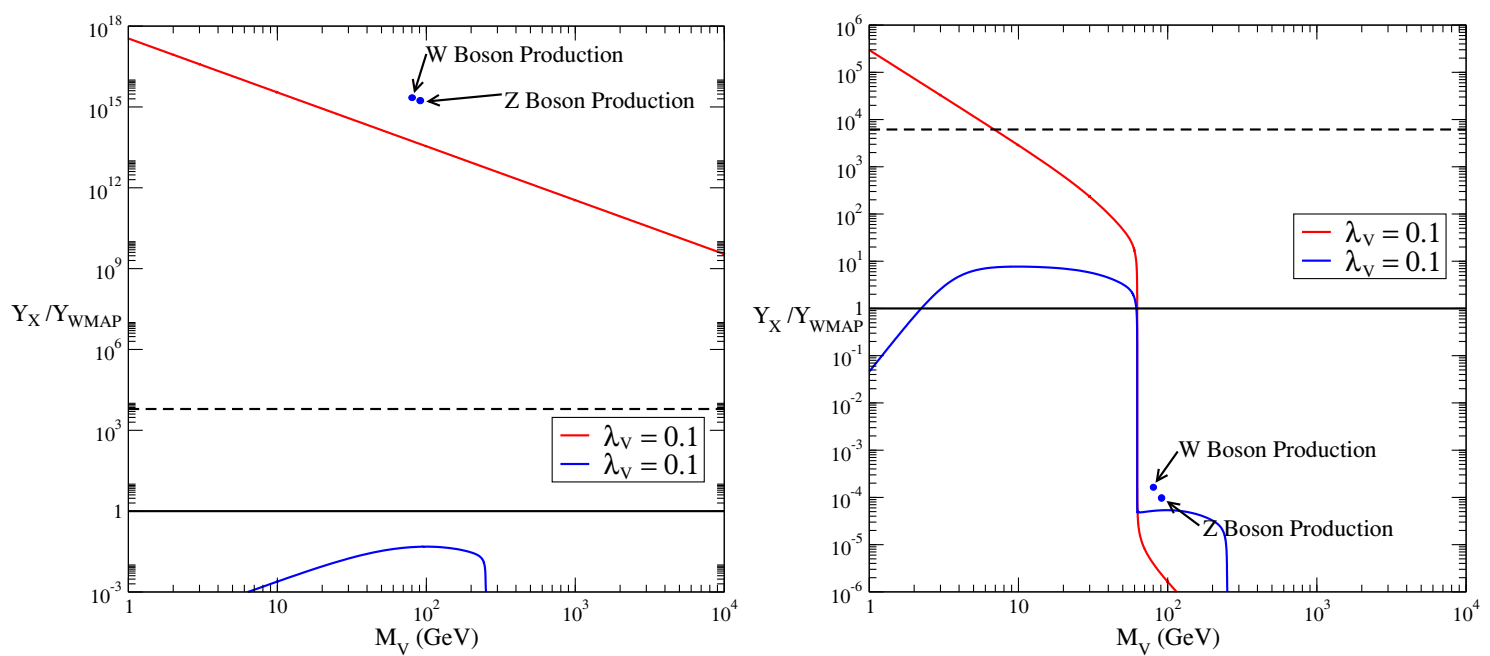

Figure 6. Generated comoving energy density $\Upsilon$ in vector bosons $V_{\mu}$ (normalized to the observed dark matter comoving energy density) as a function of the vector boson mass $M_{V}$ in the perfectly elastic collision limit (LEFT) and totally inelastic collision limit (RIGHT) for $\gamma_{w}=10^{8}$, $l_{w}=15 / T_{\mathrm{EW}}$ and $T_{\mathrm{EW}}=100 \mathrm{GeV}$. Red line: production in the presence of a direct tree-level coupling between vector bosons and Higgs (3.13). Blue line: production for a tree-level effective coupling (3.17), for $\lambda_{h s}=1$ and $M_{s}=500 \mathrm{GeV}$. The solid-black line corresponds to the observed dark matter comoving energy density, and the dashed-black line corresponds to the maximum possible generated comoving number density $\left(\mathcal{E}=E_{w}\right)$.

Vector boson effective Higgs-portal particle production is shown in figure 6, resulting in a very suppressed particle production with respect to the case in which the vector bosons and the Higgs couple directly at tree-level, specially for very elastic collisions. From figure 6 it is also clear that backreaction is most important for direct vector boson particle production (for which the production estimate yields $\mathcal{E} \gg E_{w}$ ).

\subsection{Backreaction and relative efficiency}

Clearly, for the present analysis of particle production to be physically meaningful it must be assumed that the total energy of the produced particles is less than the energy contained in the background field configuration $h(z, t)$. Moreover, when the energy of the produced particles starts being comparable to the energy of the background field we expect backreaction on $h(z, t)$ due to the particle production to be important. Then, in order for the previous analysis to be reliable, it is needed

$$
\left.\frac{\mathcal{E}}{A}\right|_{X} \ll \frac{E_{w}}{A}=\frac{2}{3} v_{T}^{2} \frac{\gamma_{w}}{l_{w}}
$$

As it has been shown in the previous section, for fermion or vector boson particle production the previous condition (3.18) is not satisfied, and in some cases even $\mathcal{E} \gg E_{w}$ is obtained (figure 6 LEFT), signaling the extreme importance of backreaction in those scenarios.

Since incorporating backreaction into the present analysis of particle production is extremely difficult and lies beyond the scope of this paper, we simply note that the relative 

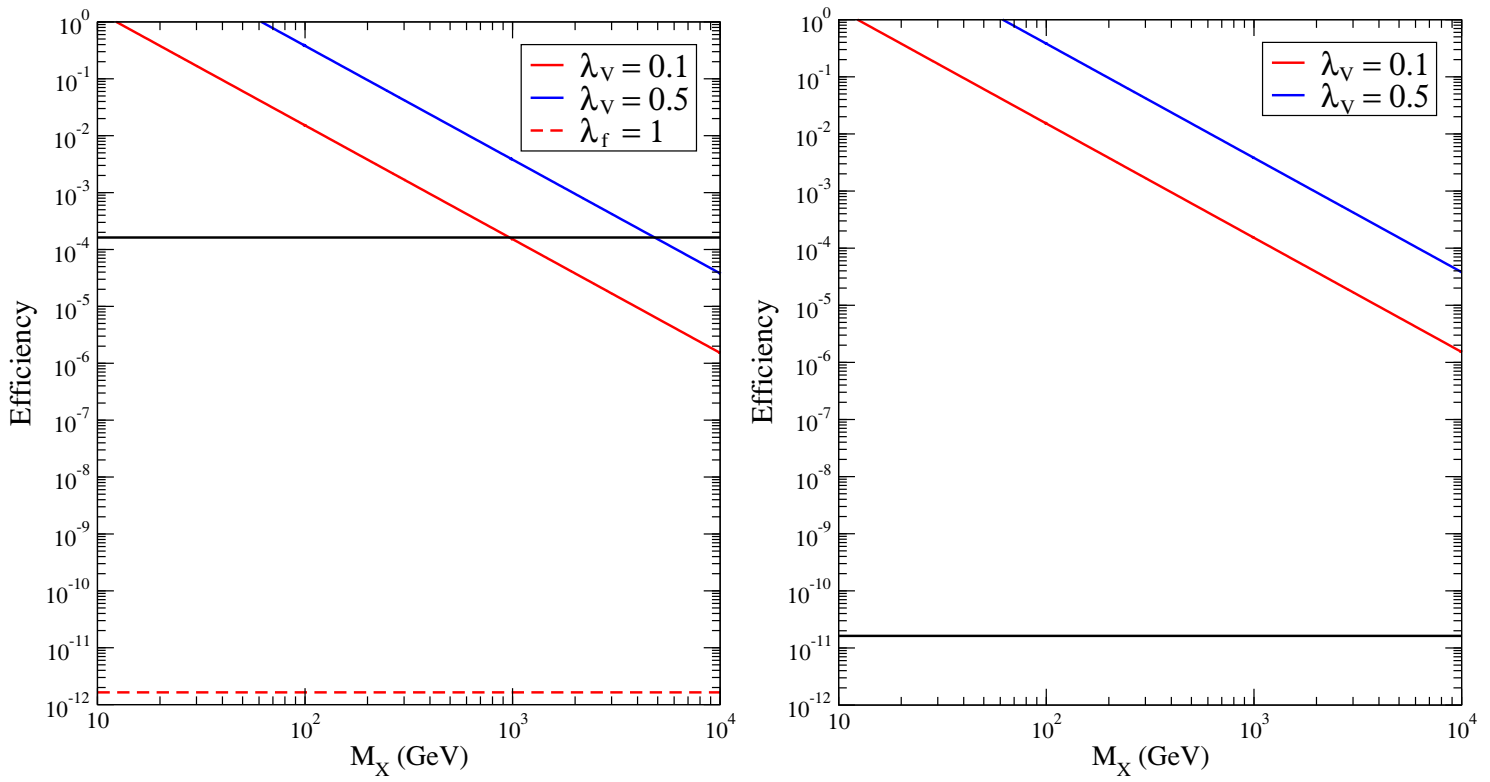

Figure 7. Efficiency of vector boson (solid lines) and fermion (dashed line) particle production (scalars are too inefficiently produced to be shown) for a perfectly elastic collision, normalized to the most efficiently produced particles (in this case $W_{\mu}$ and $Z_{\mu}$ ) and to the energy contained in the bubble walls, for $\gamma_{w}=10^{8}$ (LEFT) and $\gamma_{w}=10^{15}$ (RIGHT), $l_{w}=15 / T_{\mathrm{EW}}$ and $T_{\mathrm{EW}}=100 \mathrm{GeV}$. The solid-black line corresponds to the observed dark matter comoving energy density (normalized to the energy contained in the bubble walls).

efficiency in particle production for the different species in the present analysis should be roughly correct even when backreaction is important. Then, an estimate of the particle production in cases where some of the species are very efficiently produced may be obtained just by normalizing the production to the total energy in the bubble walls. For very elastic bubble collisions, it has been shown in section 3.3 that production of $W_{\mu}$ and $Z_{\mu}$ gauge bosons is extremely efficient, which will then leave very little energy left in the bubble walls for producing other particle species. The relative efficiencies (defined as ratios of energy in produced particles) of the different species for a perfectly elastic collision, normalized to the energy contained in the bubble walls (assuming that most of the available energy goes into producing $W_{\mu}$ and $Z_{\mu}$ ) is shown in figure 7 . A good estimate of the non-thermally generated comoving energy density (per particle species $\alpha$ ) in this case may then be given by

$$
\left.\Upsilon_{\alpha} \simeq \frac{20}{\sqrt{\pi g_{*}}} \frac{1}{M_{\mathrm{Pl}} T_{\mathrm{EW}}} \frac{\beta}{H} \frac{\mathcal{E}}{A}\right|_{\alpha}\left(\left.\frac{\mathcal{E}}{A}\right|_{W_{\mu}}\right)^{-1} \frac{E_{w}}{A}
$$

The fact that this is a reliable estimate of the particle production efficiency for the case of very elastic collisions is due to the high- $p^{2}$ modes of the bubble wall carrying almost all the energy of the bubble wall. The energy carried by the high- $p^{2}$ modes will then mostly go into vector boson production (their production efficiency at high $p^{2}$ is much larger than fermionic or scalar ones), result that holds even without incorporating backreaction into the analysis. 
On the other hand, for very inelastic collisions the results from the previous section show that particle production is only effective for light particles $\left(M_{X} \lesssim m_{h} / 2\right)$. Therefore, production of $W_{\mu}$ and $Z_{\mu}$ will be very suppressed in this case, along with any other heavy particle, and most of the available energy will go into production of SM fermions (mainly bottom quarks) and (possibly) new light scalars or fermions with sizable couplings to the Higgs.

\section{Non-thermal multi-TeV WIMP dark matter}

In this section we focus on the case of relatively heavy dark matter, $M_{X} \gtrsim \mathrm{TeV}$, and explore the conditions under which the amount of non-thermally produced heavy dark matter can end-up accounting for a sizable part of the observed dark matter relic density (dark matter may nevertheless still have a thermal component coming from the usual freeze-out process). The first condition is clearly that bubble collisions have to be fairly elastic: it has been shown in sections 3 that for very inelastic bubble collisions only light $\left(M_{X} \lesssim m_{h} / 2\right)$ particles are efficiently produced, while heavy particle production is extremely suppressed. Since fast thermalization of light species after the EW phase transition seems unavoidable, ${ }^{8}$ for very inelastic bubble collisions either dark matter is not efficiently produced or it thermalizes immediately after the end of the EW phase transition, not having any influence on the subsequent evolution of the Universe.

For very elastic bubble collisions, the analysis from sections 3 and 3.4 shows that electroweak gauge bosons $W_{\mu}$ and $Z_{\mu}$ are most efficiently produced, and the relative production efficiency of heavy fermions and scalars is too low (for them to be able to account for a sizable part of the observed dark matter relic abundance, see figure 7). This leaves heavy vector bosons with a direct coupling to the Higgs field as the only viable candidate for non-thermally produced dark matter during the EW phase transition.

In the following we perform an analysis of heavy vector boson dark matter coupled to the Higgs, including an overview of thermal freeze-out and direct detection constrains from XENON100 [33] (see [34, 35] for more details), and a comparison between the amount of non-thermally produced dark matter and the amount of dark matter produced through thermal freeze-out. We also study the evolution of the non-thermally produced dark matter component after the EW phase transition.

\subsection{Higgs-vector dark matter interplay}

Consider a vector boson dark matter candidate with mass $M_{V}$ and a tree-level coupling to the Higgs [34, 36, 37],

$$
\mathcal{L}_{V}=\frac{1}{2} M_{V}^{2} V_{\mu} V_{\mu}+\lambda_{V} v_{T} h V_{\mu} V_{\mu}
$$

This coupling mediates the dark matter annihilation into Standard Model particles, as well as the elastic scattering on nucleons relevant for dark matter direct detection. Concerning the former process, the Higgs boson can mediate annihilation of dark matter into

\footnotetext{
${ }^{8}$ Dark matter may be coupled to the Higgs weakly enough as to avoid thermalization, however in that case we find it is not produced in sufficient quantities to make up for the observed relic abundance. For a discussion of the asymmetric dark matter case, see apppendix A.
} 
electroweak gauge bosons (for heavy dark matter they are the most important annihilation channel) through the couplings

$$
\frac{h}{v_{T}}\left(2 M_{W}^{2} W_{\mu}^{+} W_{\mu}^{-}+M_{Z}^{2} Z_{\mu} Z_{\mu}\right)
$$

The spin-averaged amplitude squared for the annihilation process $V_{\mu} V_{\mu} \rightarrow W_{\mu}^{+} W_{\mu}^{-}$in the limit $s \gg m_{h}^{2}$ is given by

$$
\left|\overline{\mathcal{M}}_{V V \rightarrow W / Z, W / Z}\right|^{2} \approx \frac{2}{3} \lambda_{V}^{2}\left(\frac{s^{2}}{4 M_{V}^{4}}-\frac{s}{M_{V}^{2}}+3\right)
$$

Given (4.3), the thermally averaged annihilation cross section is given by

$$
\langle\sigma v\rangle_{V V \rightarrow W / Z, W / Z}=\frac{z \lambda_{V}^{2}}{192 \pi M_{V}^{2} K_{2}(z)^{2}} \int_{4}^{\infty} d x \sqrt{x-4} K_{1}(\sqrt{x} z)\left(\frac{x(x-4)}{4}+3\right)
$$

where $z=M_{V} / T$, and $K_{1}(z), K_{2}(z)$ are Bessel functions. For $z \gg 1,(4.4)$ reduces to

$$
\langle\sigma v\rangle_{V V \rightarrow W / Z, W / Z} \approx \frac{\lambda_{V}^{2}}{16 \pi M_{V}^{2}}
$$

The thermal cross section giving rise to the observed value of the relic density $\langle\sigma v\rangle_{\mathrm{WMAP}} \approx 2.6 \cdot 10^{-9} \mathrm{GeV}^{-2}$ corresponds, for heavy dark matter $M_{V} \gg m_{h}$ and using (4.5), to

$$
\left[\frac{\lambda_{V}}{M_{V}(\mathrm{TeV})}\right]_{\mathrm{WMAP}} \approx 0.3
$$

Turning now to dark matter direct detection, the spin-averaged amplitude squared for Higgs-mediated dark matter elastic scattering on nucleons reads

$$
\left|\overline{\mathcal{M}}_{V N \rightarrow V N}\right|^{2}=\frac{8 \lambda_{V}^{2} f_{N}^{2} m_{N}^{2}}{3\left(t-m_{h}^{2}\right)^{2}}\left(2+\frac{\left(M_{V}^{2}-\frac{t}{2}\right)}{M_{V}^{2}}\right)\left(2 m_{N}^{2}-\frac{t}{2}\right) \approx \frac{16 \lambda_{V}^{2} f_{N}^{2} m_{N}^{4}}{m_{h}^{4}}
$$

Here, $m_{N} \approx 0.939 \mathrm{GeV}$ is the proton/neutron mass and $f_{N}$ is the effective Yukawa coupling of the Higgs to nucleons which, following [35], we take $f_{N}=0.326$ based on the lattice estimate in [38] (see also [39]). In the last step we have taken the limit $t \ll m_{N}^{2}, m_{h}^{2}, M_{V}^{2}$. The elastic scattering cross section then reads

$$
\sigma_{V N \rightarrow V N} \approx \frac{\lambda_{V}^{2} f_{N}^{2} m_{N}^{4}}{\pi M_{V}^{2} m_{h}^{4}} \approx 4.2 \cdot 10^{-44} \mathrm{~cm}^{2}\left[\frac{\lambda_{V}}{M_{V}(\mathrm{TeV})}\right]^{2}
$$

On the other hand, the XENON100 bound on the dark matter elastic scattering cross section on nucleons, for $M_{V} \gtrsim \mathrm{TeV}$ is approximately

$$
\sigma_{V N \rightarrow V N}<M_{V} \cdot 2.2 \cdot 10^{-44} \mathrm{~cm}^{2}
$$

Therefore, (4.6) and (4.9) leave a sizable window in the parameter space $\left(M_{V}, \lambda_{V}\right)$ for which the dark matter abundance obtained via thermal freeze-out is significantly smaller than the observed dark matter relic density, and still the value of $\lambda_{V}$ (as a function of $M_{V}$ ) is below the XENON100 bound (as shown in figure 8). 


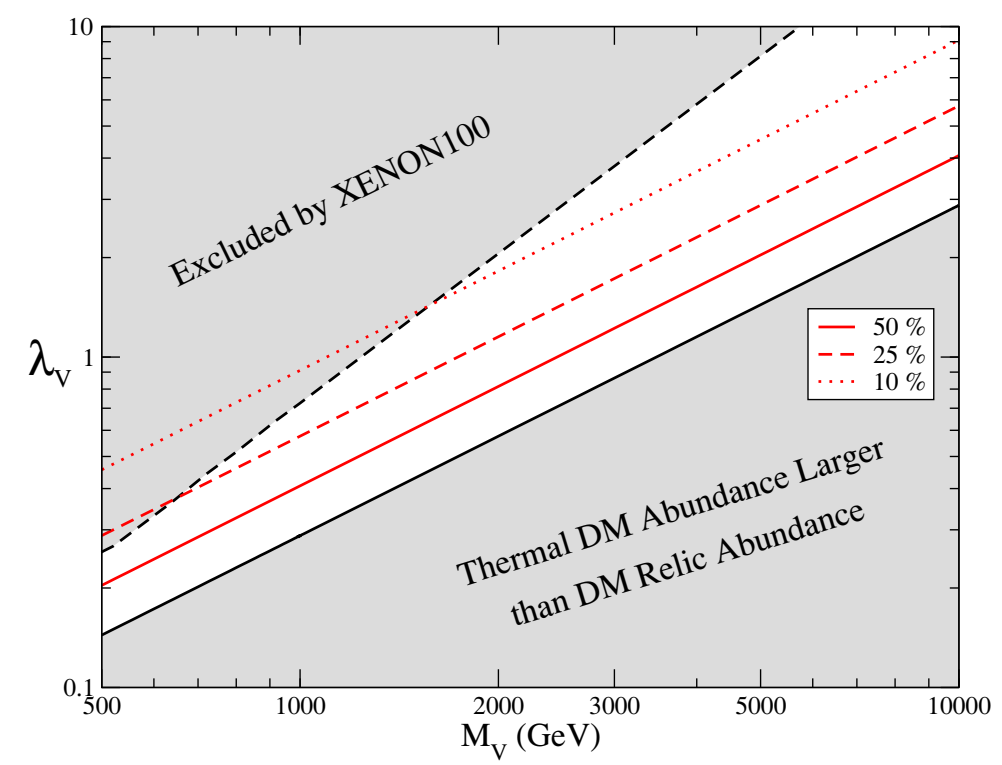

Figure 8. The dashed-black line corresponds to the limits on $\lambda_{V}$ from XENON100 (4.9). The solid-black line corresponds to the value of $\lambda_{V}$ for which the observed DM relic density is obtained via thermal freeze-out (4.6): below it the thermal DM density is larger than the observed DM relic density (and thus this region is excluded). Above, the thermal DM density is only a fraction of the observed DM relic density, and the red lines show the percentage of relic density accounted for by the thermal density.

\subsection{Fate of non-thermally produced vector dark matter}

Given the results from the previous section (summarized in figure 8), it is fair to ask if, in the region of $\left(M_{V}, \lambda_{V}\right)$ parameter space in which the thermal component is not enough to account for the observed dark matter relic density, dark matter produced non-thermally at the EW phase transition could account for the extra needed amount. Using the results from production efficiency of heavy vector boson dark matter obtained in sections 3.3 and 3.4, we show in figure 9 the value of $\lambda_{V}$ (as a function of $M_{V}$ ) for which the amount of non-thermal vector boson production equals the observed dark matter relic density (dashed-blue line). Then, for values of $\lambda_{V}$ above the thermal cross section giving the observed relic density $\langle\sigma v\rangle_{\mathrm{WMAP}}$, non-thermal production of heavy vector bosons is so efficient as to generate amounts of dark matter much larger than the observed dark matter relic density.

Assuming that at the time of the EW phase transition vector boson dark matter is already frozen-out $\left(T_{\mathrm{fo}} \simeq M_{V} / 20>T_{\mathrm{EW}}\right.$ ), we can study the evolution of the non-thermally generated dark matter abundance via a simple Boltzmann equation in which the comoving dark matter number density $Y$ fulfills $Y(z) \gg Y_{E Q}(z)$ (with $Y_{E Q}(z)$ being the equilibrium comoving number density), yielding

$$
\frac{d Y}{d z}=-\alpha \frac{\langle\sigma v\rangle M_{\mathrm{Pl}} M_{V}}{z^{2}} Y(z)^{2} \longrightarrow \frac{d y}{d z}=-\frac{1}{z^{2}} y^{2}(z)
$$

with $\alpha=\left(4 \pi^{2} \sqrt{\xi g_{*}}\right) / 45 \simeq 2.642\left(g_{*} \sim 100\right.$ being the number of relativistic degrees of freedom in the thermal plasma and $\left.\xi \equiv 90 /\left(32 \pi^{3}\right)\right), M_{\mathrm{Pl}}=1.2 \times 10^{19} \mathrm{GeV}$ and $y(z)=$ 


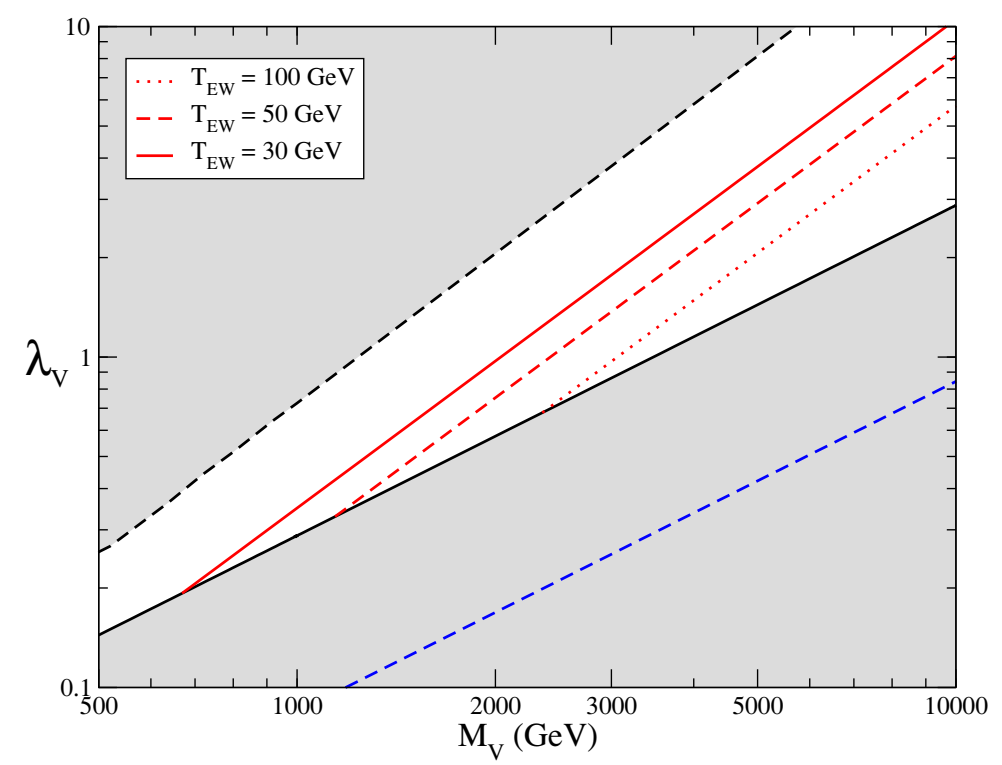

Figure 9. Black lines are the same as in figure 8. The dashed-blue line corresponds to the value of $\lambda_{V}$ needed for the non-thermally produced energy density in vector bosons (with a direct coupling to the Higgs) to be equal to the DM relic density, for $\gamma_{w}=10^{8}$. The red lines show the values of $\lambda_{V}$ yielding the "non-thermal" cross section (4.13) (for which the final dark matter abundance, taking into account its evolution after non-thermal production, corresponds to the observed dark matter relic density) for several values of $T_{\mathrm{EW}}$.

$\alpha\langle\sigma v\rangle M_{\mathrm{Pl}} M_{V} Y(z)$. Integration of (4.10) for $z>z_{\mathrm{EW}}$ yields

$$
\frac{1}{y(z)}-\frac{1}{y\left(z_{\mathrm{EW}}\right)}=\frac{1}{z_{\mathrm{EW}}}-\frac{1}{z} \longrightarrow \frac{1}{y(\infty)}=\frac{1}{z_{\mathrm{EW}}}+\frac{1}{y\left(z_{\mathrm{EW}}\right)}
$$

Then, given the fact that non-thermal vector boson dark matter production is much larger than the observed relic density in the $\left(M_{V}, \lambda_{V}\right)$ region of interest, we can take the limit $y(\infty) \ll y\left(z_{\mathrm{EW}}\right)$, obtaining

$$
y(\infty) \simeq z_{\mathrm{EW}}
$$

From (4.12), we immediately obtain that the value of the annihilation cross section that will yield the observed dark matter relic density once the non-thermally generated dark matter evolves after the EW phase transition is simply given by

$$
\langle\sigma v\rangle=\langle\sigma v\rangle_{\mathrm{WMAP}} \frac{T_{\mathrm{fo}}}{T_{\mathrm{EW}}}
$$

The red lines in figure 9 show the values of $\lambda_{V}$ yielding the correct "non-thermal" annihilation cross section (4.13) for several values of $T_{\mathrm{EW}}$.

This analysis shows that non-thermal production of multi-TeV vector boson dark matter at the EW phase transition (in $\left(M_{V}, \lambda_{V}\right)$ parameter space in which the amount of dark matter yielded by thermal freeze-out is not enough to account for the observed dark matter relic density) is efficient as to generate a dark matter amount much larger than the observed relic density. This results in a reactivation of thermalization processes that lead to partial 


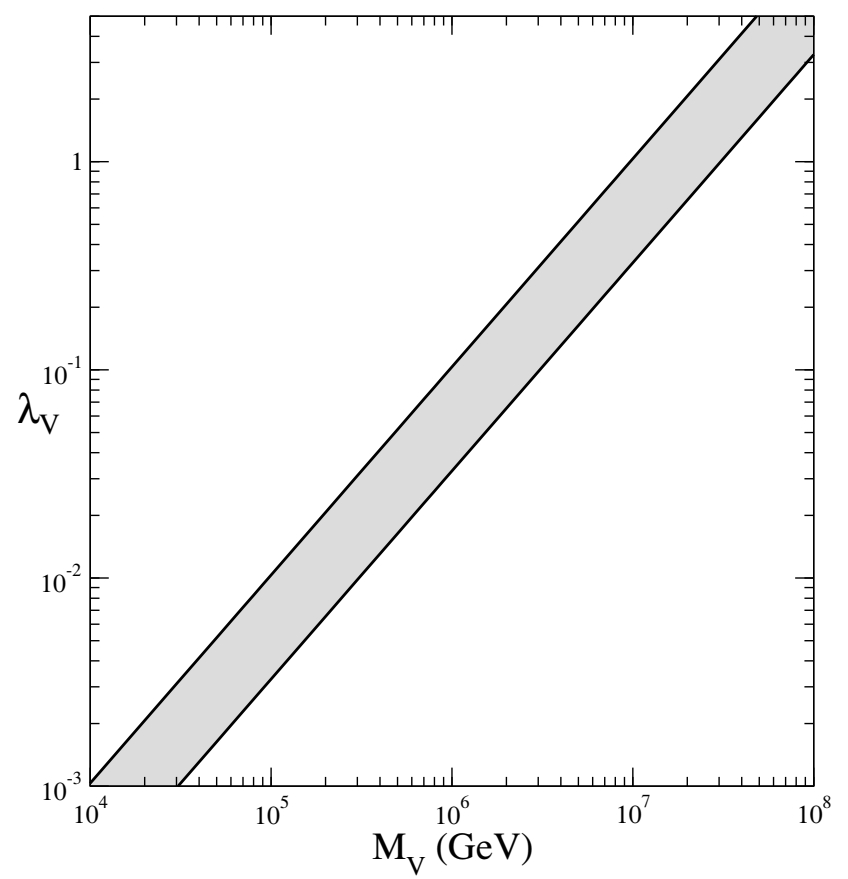

Figure 10. Region in the $\left(M_{V}, \lambda_{V}\right)$ parameter space for which non-thermal $V_{\mu}$ production yields the observed dark matter relic density for $l_{w}=15 / T_{\mathrm{EW}}\left(\right.$ with $\left.T_{\mathrm{EW}}=100 \mathrm{GeV}\right)$ and $\gamma_{w}=10^{14}-10^{15}$.

wash-out of the non-thermally generated dark matter (wash-out is not complete due to the reactivation happening for $T<T_{\mathrm{EW}}<T_{\mathrm{fo}}$ ), meaning that multi-TeV dark matter may have a thermal spectrum despite a large fraction of it having been produced non-thermally at the EW phase transition. As shown in figure 9, in the presence of these non-thermally produced WIMPs, the relation between mass and coupling giving rise to the observed dark matter relic density gets modified with respect to the usual thermal freeze-out scenario, leading to better detection prospects in the multi-TeV region for future dark matter direct detection experiments.

\section{Baby-zillas: super-heavy dark matter from the EW phase transition}

In this section we study the production of super-heavy dark matter with a mass $M_{X}$ satisfying $M_{\mathrm{GUT}} \gg M_{X} \gg v_{\mathrm{EW}}$ in the bubble collisions at the end of a very strong EW phase transition. We call these dark matter particles baby-zillas because of many similarities (but smaller mass) to the WIMP-zilla scenario [7-10].

From figure 7 , it can be inferred that for $\gamma_{w} \sim 10^{14}-10^{15}$ non-thermal heavy vector boson production in elastic bubble collisions can be so efficient as to generate the observed dark matter relic density even for very large dark matter masses $M_{V} \sim 10^{6}-10^{8} \mathrm{GeV}$ and perturbative values of the coupling $\lambda_{V}$. Using (3.19), we plot in figure 10 the region in parameter space $\left(M_{V}, \lambda_{V}\right)$ for which non-thermal $V_{\mu}$ production directly yields the observed dark matter relic density. 


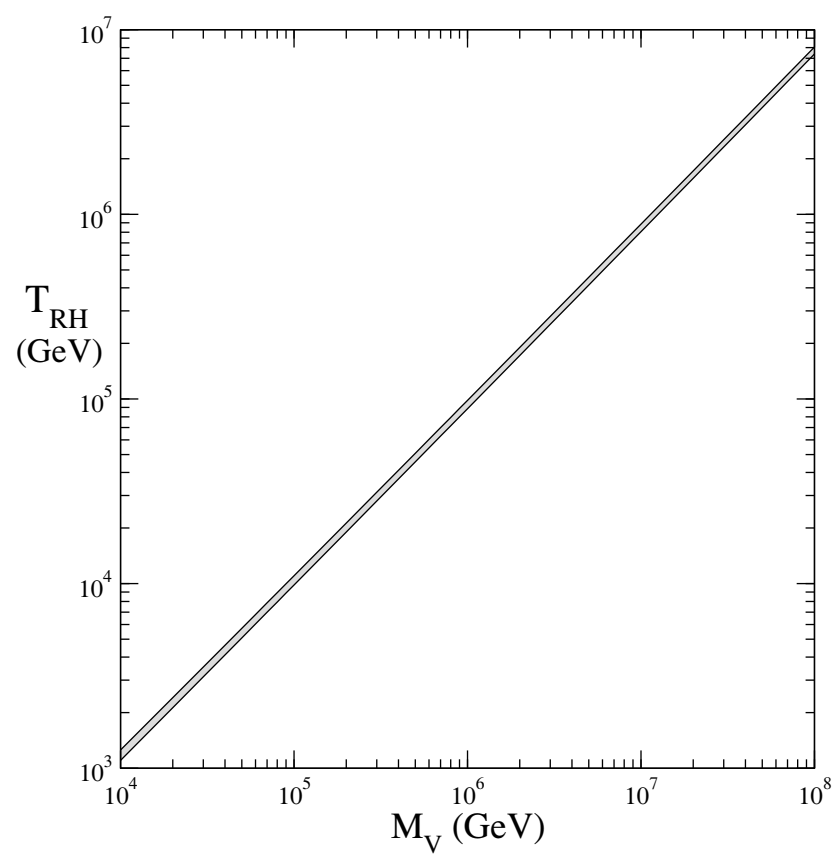

Figure 11. Bounds on the Reheating temperature after inflation for the requirement that dark matter never reaches thermal equilibrium after inflation, namely $T_{\mathrm{RH}} \leq T_{\mathrm{fo}}$, as a function of the dark matter mass $M_{V}$, and assuming $\lambda_{V}\left(M_{V}\right)$ for which non-thermal production yields the observed relic abundance (as shown in figure 10).

\subsection{Bounds on the reheating temperature after inflation}

A stable particle with mass $M_{V} \sim 10^{5}-10^{8} \mathrm{GeV}$ would yield a much larger relic abundance than the observed DM relic density. were it in thermal equilibrium at some stage after inflation. For such a massive species, the annihilation cross is always smaller than the one needed to yield the observed DM relic density through thermal freeze-out. It is then needed that this particle species never reached thermal equilibrium after the end of inflation. This sets an upper bound on the reheating temperature after inflation, specifically $T_{\mathrm{RH}}<T_{\text {fo }}$ (with $T_{\mathrm{fo}}$ being the temperature below which the particle is decoupled from the thermal plasma). For a heavy vector boson $V_{\mu}$ annihilating into $\mathrm{SU}(2)$ gauge bosons (the most important annihilation channel in this case) through the Higgs, $T_{\mathrm{fo}}$ satifies

$$
\frac{M_{V}}{T_{\mathrm{fo}}} \simeq 20.4+\log \left(\frac{M_{V}}{100 \mathrm{GeV}}\right)+\log \left(\frac{\langle\sigma v\rangle}{10^{-9} \mathrm{GeV}^{-2}}\right)
$$

where the thermally averaged annihilation cross section $\langle\sigma v\rangle$ is given by (4.4). In figure 11 we plot the minimum value of $z$ (corresponding to the maximum allowed value of the reheating temperature $T_{\mathrm{RH}}$ ) as a function of the mass $M_{V}$ for the range of $\lambda_{V}$ values giving rise to the observed dark matter relic abundance for $\gamma_{w}=10^{14}-10^{15}$ (see figure 10). We see that the upper bound on $T_{\mathrm{RH}}$ is relatively insensitive to the precise value of $\gamma_{w}$, and roughly scales as $T_{\mathrm{RH}}^{\max } \sim M_{V} / 10$. 


\section{Conclusions}

Dark matter may have been efficiently produced at the end of a first order EW phase transition if it has a large coupling to the Higgs field. In this paper we investigated the conditions for this non-thermal production mechanism to account for most of dark matter in the Universe. We considered scalar, fermion and vector dark matter coupled to the SM through the Higgs (either via a direct, tree-level interaction or an effective Higgs-portal coupling), and found that production of vector bosons directly coupled to the Higgs is most efficient, while for scalar and fermions most of the energy stored in the bubble walls is bound to be released into production of SM particles. This analysis singles out vector dark matter in the present context.

For very inelastic bubble collisions only dark with $M_{X} \lesssim 100 \mathrm{GeV}$ can be efficiently produced, while production of heavier dark matter is extremely suppressed. Unfortunately, for a dark matter mass in this range, we did not find a way to avoid subsequent thermalization and the wash-out of the non-thermal component, and therefore in this case dark matter production at the EW phase transition is irrelevant. The situation is quite different for highly elastic bubble collisions. In that case, dark matter with $M_{X} \gg 100 \mathrm{GeV}$ can be efficiently produced for the so-called runaway bubbles, that expand with a very large $\gamma$-factor.

We have identified two scenarios where wash-out of dark matter produced at the EW phase transition can be naturally avoided. One has dark matter in the multi-TeV range, which makes it possible for non-thermally produced dark matter to remain out of thermal equilibrium after the EW phase transition. We determined the region in the parameter space of dark matter mass and coupling to the Higgs where the correct relic abundance is reproduced. For a given mass, the coupling has to be larger than in the usual thermal freeze-out scenario for Higgs portal dark matter, which can be especially relevant for direct detection searches, as it opens the possibility of detecting a signal from multi-TeV nonthermal dark matter in the near future by XENON100 and LUX experiments. The other scenario is baby-zilla dark matter with $M_{X} \sim 10^{6}-10^{8} \mathrm{GeV}$. Surprisingly enough, such super-heavy dark matter can be produced in important quantities at the end of a strongly first-order EW phase transition, provided the dark matter coupling to the Higgs is large, and the $\gamma$ factor of the bubble walls is near its maximal value of $\gamma_{w} \sim 10^{15}$. In order for the baby-zillas to be a viable dark matter candidate, they must have never reached thermal equilibrium, which then constrains the reheating temperature after inflation in this scenario.

\section{Acknowledgments}

We specially thank Francesco Riva for very useful discussions and collaboration in the early stages of this work, and also Thomas Konstandin, Michel Tytgat, Yann Mambrini, Stephan Huber and Stephen West for discussions and comments. The work of J.M.N. is supported by the Science Technology and Facilities Council (STFC) under grant number $\mathrm{ST} / \mathrm{J} 000477 / 1$. 


\section{A Asymmetric dark matter production}

We now explore the possibility of asymmetric dark matter production during the EW phase transition, together with the viability of this mechanism as a way to avoid wash-out of non-thermal production for relatively light dark matter (and any other light species in general).

For multi-component dark matter $\left(X=X_{\alpha}\right)$, an asymmetry in the number densities of $X_{\alpha}$ and $\bar{X}_{\alpha}$ may be generated during the particle production. We will analyze in detail below the generation of this asymmetry for scalars $\left(X_{\alpha}=S_{\alpha}\right)$. Then, in section A.2 we study the evolution of the generated asymmetries after the EW phase transition.

\section{A.1 Decay asymmetries: producing a dark matter asymmetry}

Let us consider a set of $N_{i}$ real scalars $h_{i}$ (that includes the field(s) involved in the EW phase transition) coupled to a set of $N_{\alpha}$ complex scalars $S_{\alpha}$ via a trilinear interaction. The relevant part of the lagrangian is

$$
-\Delta \mathcal{L}=m_{\alpha}^{2} S_{\alpha}^{*} S_{\alpha}+C_{i \alpha \beta} h_{i} S_{\alpha}^{*} S_{\beta}+V\left(h_{i}\right)
$$

where by hermiticity $C_{i \alpha \beta}=C_{i \beta \alpha}^{*}$ (it follows that $C_{i \alpha \alpha}$ are real, but $C_{i \alpha \beta}$ with $\alpha \neq \beta$ can be complex), and the mass matrix for the scalars $S_{\alpha}$ is taken to be diagonal without loss of generality. We also consider a possible term $\mu_{i j} h_{i} h_{j}^{2}$ appearing in $V\left(h_{i}\right)$. The lagrangian (A.1) incorporates a $Z_{2}$ symmetry that makes the lightest of the scalars $S_{\alpha}$ stable, which may then be a suitable dark matter candidate. In order for an asymmetry in the production of $S_{\alpha}$ and $S_{\alpha}^{*}$ to be generated, we need a nonzero value for

$$
\left|\mathcal{M}\left(h_{i} \rightarrow S_{\alpha}^{*} S_{\beta}\right)\right|^{2}-\left|\mathcal{M}\left(h_{i} \rightarrow S_{\beta}^{*} S_{\alpha}\right)\right|^{2}
$$

At tree level

$$
\begin{aligned}
& \mathcal{M}^{\text {Tree }}\left(h_{i} \rightarrow S_{\alpha}^{*} S_{\beta}\right)=C_{i \alpha \beta} \\
& \mathcal{M}^{\text {Tree }}\left(h_{i} \rightarrow S_{\beta}^{*} S_{\alpha}\right)=C_{i \alpha \beta}^{*}
\end{aligned} \quad \Rightarrow \quad\left|\mathcal{M}\left(h_{i} \rightarrow S_{\alpha}^{*} S_{\beta}\right)\right|^{2}=\left|\mathcal{M}\left(h_{i} \rightarrow S_{\beta}^{*} S_{\alpha}\right)\right|^{2}=\left|C_{i \alpha \beta}\right|^{2}
$$

and there is no asymmetry generated. At 1-loop we include the 1PI diagrams shown in figure 12. Their contribution to the 1-loop decay amplitude is

$$
\begin{aligned}
& \mathcal{M}^{1 \mathrm{~L}}\left(h_{i} \rightarrow S_{\alpha}^{*} S_{\beta}\right)=-\frac{1}{16 \pi^{2}} \sum_{j, \gamma, \delta}\left(C_{i \gamma \delta} C_{j \alpha \gamma} C_{j \delta \beta} I_{T}+\mu_{i j} C_{j \alpha \delta} C_{j \delta \beta} \tilde{I}_{T}\right) \\
& \mathcal{M}^{1 \mathrm{~L}}\left(h_{i} \rightarrow S_{\beta}^{*} S_{\alpha}\right)=-\frac{1}{16 \pi^{2}} \sum_{j, \gamma, \delta}\left(C_{i \gamma \delta}^{*} C_{j \alpha \gamma}^{*} C_{j \delta \beta}^{*} I_{T}+\mu_{i j} C_{j \alpha \delta}^{*} C_{j \delta \beta}^{*} \tilde{I}_{T}\right)
\end{aligned}
$$

where the integrals $I_{T}$ and $\tilde{I}_{T}$ correspond to

$$
\begin{aligned}
I_{T} & =\frac{-i}{\pi^{2}} \int d^{4} k \frac{1}{\left(k^{2}-m_{\gamma}^{2}\right)\left((k+p)^{2}-m_{\delta}^{2}\right)\left(\left(k-k_{2}\right)^{2}-m_{j}^{2}\right)} \\
\tilde{I}_{T} & =\frac{-i}{\pi^{2}} \int d^{4} k \frac{1}{\left(k^{2}-m_{j}^{2}\right)\left((k+p)^{2}-m_{j}^{2}\right)\left(\left(k-k_{2}\right)^{2}-m_{\delta}^{2}\right)}
\end{aligned}
$$




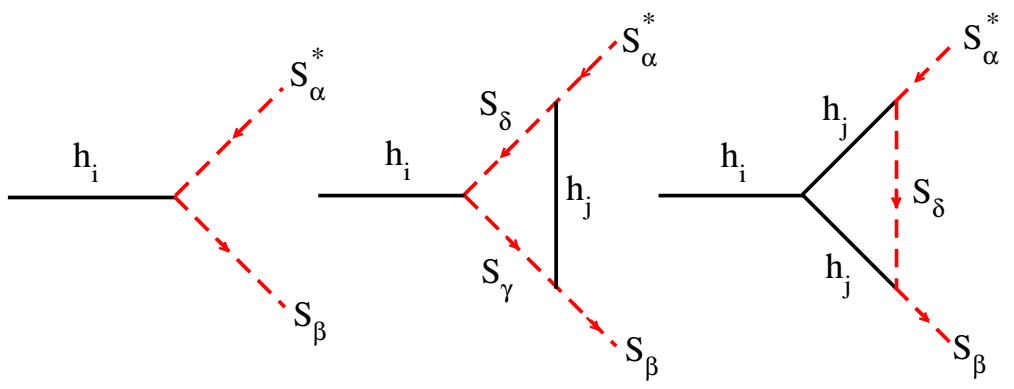

Figure 12. Tree-level and 1PI 1-loop contributions to the decay $h_{i} \rightarrow S_{\alpha}^{*} S_{\beta}$

and can be computed in terms of the usual Passarino-Veltman 3-point scalar loop integral $C_{0}$. The leading order difference between $\left|\mathcal{M}\left(i \rightarrow \alpha^{*} \beta\right)\right|^{2}$ and $\left|\mathcal{M}\left(i \rightarrow \alpha \beta^{*}\right)\right|^{2}$ is due to the interference between the tree level and 1-loop decay amplitudes. We obtain

$$
\begin{gathered}
\left|\mathcal{M}\left(h_{i} \rightarrow S_{\alpha}^{*} S_{\beta}\right)\right|^{2}-\left|\mathcal{M}\left(h_{i} \rightarrow S_{\beta}^{*} S_{\alpha}\right)\right|^{2}= \\
\frac{1}{4 \pi^{2}} \sum_{j, \gamma, \delta}\left\{\mu_{i j} \operatorname{Im}\left[C_{i \alpha \beta}^{*} C_{j \alpha \delta} C_{j \delta \beta}\right] \operatorname{Im}\left[\tilde{I}_{T}\right]+\operatorname{Im}\left[C_{i \alpha \beta}^{*} C_{i \gamma \delta} C_{j \alpha \gamma} C_{j \delta \beta}\right] \operatorname{Im}\left[I_{T}\right]\right\}
\end{gathered}
$$

An interplay between "weak" and "strong" phases (complex couplings and imaginary part of a 1-loop integral due to particles in the loop going on-shell) is then needed to get CP violation in the decay. For $N_{i}=1(i=j=1)$ both terms on the right-hand side of (A.6) will vanish for $N_{\alpha}<3$ but may be zonzero for $N_{\alpha} \geq 3$. For the case of two fields $h_{i}\left(N_{i}=2\right)$, already with two scalars $\left(N_{\alpha} \geq 2\right)$ it is possible to obtain an asymmetry.

To obtain the total combined production of $S_{\alpha}$ and $S_{\alpha}^{*}$ particles, we will just consider the tree level contribution to $h_{i} \rightarrow S_{\alpha}^{*} S_{\alpha}, h_{i} \rightarrow S_{\alpha}^{*} S_{\beta}$ and $h_{i} \rightarrow S_{\beta}^{*} S_{\alpha}$. We then get

$$
\operatorname{Im}\left[\tilde{\Gamma}^{(2)}(\chi)\right]_{\alpha}=\left|C_{i \alpha \alpha}\right|^{2} \int d \Pi_{\alpha \alpha}+\left|C_{i \alpha \beta}\right|^{2} \int d \Pi_{\alpha \beta}
$$

with

$$
\begin{gathered}
\int d \Pi_{\alpha \alpha}=\frac{1}{8 \pi} \sqrt{1-\frac{4 m_{\alpha}^{2}}{\chi}} \Theta\left(\chi-4 m_{\alpha}^{2}\right) \\
\int d \Pi_{\alpha \beta}=\frac{1}{8 \pi} \sqrt{1-2 \frac{m_{\alpha}^{2}+m_{\beta}^{2}}{\chi}+\frac{\left(m_{\alpha}^{2}-m_{\beta}^{2}\right)^{2}}{\chi^{2}}} \Theta\left(\chi-\left(m_{\alpha}+m_{\beta}\right)^{2}\right)
\end{gathered}
$$

For the asymmetry in the production of $S_{\alpha}$ and $S_{\alpha}^{*}$ particles we obtain

$$
\operatorname{Im}\left[\tilde{\Gamma}^{(2)}(\chi)\right]_{\alpha}^{\mathrm{Asym}}=\left.\left(\left|\mathcal{M}\left(h_{i} \rightarrow S_{\alpha}^{*} S_{\beta}\right)\right|^{2}-\left|\mathcal{M}\left(h_{i} \rightarrow S_{\beta}^{*} S_{\alpha}\right)\right|^{2}\right)\right|_{p^{2}=\chi} \int d \Pi_{\alpha \beta}
$$

with $\left|\mathcal{M}\left(i \rightarrow \alpha^{*} \beta\right)\right|^{2}-\left|\mathcal{M}\left(i \rightarrow \alpha \beta^{*}\right)\right|^{2}$ given by (A.6). 


\section{A.2 Fate of the generated asymmetric abundance}

The asymmetric dark matter production process outlined in the previous section will generically result in asymmetries for the comoving number densities for particles and antiparticles of the different species $\Delta_{\alpha} \equiv Y_{X_{\alpha}}-Y_{X_{\alpha}^{*}} \neq 0$ at the end of the EW phase transition. Note however that the $Z_{2}$ symmetry forces the sum of the asymmetries of the different species $X_{\alpha}$ to vanish

$$
\sum_{\alpha} \Delta_{\alpha}=0
$$

After the EW phase transition, the comoving number densities for the different species $Y_{X_{\alpha}}$ will evolve according to a system of coupled Boltzmann equations. Denoting the symmetric and asymmetric part of the comoving number densities for particles and antiparticles of the different species by $\Xi_{\alpha} \equiv Y_{X_{\alpha}}+Y_{X_{\alpha}^{*}}$ and $\Delta_{\alpha}$, we can write

$$
\begin{aligned}
z H(z) \frac{d \Xi_{\alpha}}{d z}= & -\frac{s}{2}\langle\sigma v\rangle_{\alpha+\alpha^{*} \rightarrow \mathrm{SM}}\left[\Xi_{\alpha}^{2}-\Delta_{\alpha}^{2}-\left(\Xi_{\alpha}^{\mathrm{Eq}}\right)^{2}\right] \\
& -\frac{s}{2} \sum_{\beta \neq \alpha}\langle\sigma v\rangle_{\alpha+\beta^{*} \rightarrow \mathrm{SM}}\left[\Xi_{\alpha} \Xi_{\beta}-\Delta_{\alpha} \Delta_{\beta}-\Xi_{\alpha}^{\mathrm{Eq}} \Xi_{\beta}^{\mathrm{Eq}}\right] \\
& -\sum_{\beta \neq \alpha}\langle\sigma v\rangle_{\alpha+\mathrm{SM} \rightarrow \beta+\mathrm{SM}}\left[\Xi_{\alpha}-\frac{\Xi_{\alpha}^{\mathrm{Eq}} \Xi_{\beta}}{\Xi_{\beta}^{\mathrm{Eq}}}\right] \\
& -\sum_{\beta \neq \alpha} \Gamma_{\alpha \rightarrow \beta+\mathrm{SM}}\left[\Xi_{\alpha}-\frac{\Xi_{\alpha}^{\mathrm{Eq}} \Xi_{\beta}}{\left.\Xi_{\beta}^{\mathrm{Eq}}\right]}\right. \\
z H(z) \frac{d \Delta_{\alpha}}{d z}= & -\frac{s}{2} \sum_{\beta \neq \alpha}\langle\sigma v\rangle_{\alpha+\beta^{*} \rightarrow \mathrm{SM}}\left[\Delta_{\alpha} \Xi_{\beta}-\Xi_{\alpha} \Delta_{\beta}\right] \\
& -\sum_{\beta \neq \alpha}\langle\sigma v\rangle_{\alpha+\mathrm{SM} \rightarrow \beta+\mathrm{SM}}\left[\Delta_{\alpha}-\frac{\Xi_{\alpha}^{\mathrm{Eq}} \Delta_{\beta}}{\left.\Xi_{\beta}^{\mathrm{Eq}}\right]}\right. \\
& -\sum_{\beta \neq \alpha} \Gamma_{\alpha \rightarrow \beta+\mathrm{SM}}\left[\Delta_{\alpha}-\frac{\Xi_{\alpha}^{\mathrm{Eq}} \Delta_{\beta}}{\Xi_{\beta}^{\mathrm{Eq}}}\right]
\end{aligned}
$$

where $s$ is the entropy density, $z=m_{L} / T$ ( $m_{L}$ is the mass of the lightest species $\left.X_{\alpha}\right)$ and $H(z)$ is the Hubble parameter. From (A.12), if the annihilation processes are unsuppressed the symmetric comoving number densities for the various species $X_{\alpha}$ will be driven close to thermal equilibrium (the small departure from equilibrium being due to the presence of an asymmetry $\left.\Delta_{\alpha}\right)$

$$
\Xi_{\alpha} \rightarrow \sqrt{\Delta_{\alpha}^{2}+\left(\Xi_{\alpha}^{\mathrm{Eq}}\right)^{2}}
$$

Ideally, in the absence of wash-out of $\Delta_{\alpha}$, these processes would delay freeze-out and still be active for $\Delta_{\alpha} \gg \Xi_{\alpha}^{\mathrm{Eq}}$, annihilating away the symmetric part of the number density and leading to $\Xi_{\alpha} \rightarrow \Delta_{\alpha}$. However, the various processes entering (A.13) will tend to erase the asymmetries $\Delta_{\alpha}$. In particular, the process $X_{\alpha}+\mathrm{SM} \rightarrow X_{\beta}+\mathrm{SM}$ (responsible for kinetic equilibrium among the different species $X_{\alpha}$ ) and the decay process $X_{\alpha} \rightarrow X_{\beta}+$ $\mathrm{SM}$, will, if active, wash-out the asymmetries very rapidly, being also quite insensitive to 
the temperature of the Universe after the EW phase transition (the annihilation process $X_{\alpha}+X_{\beta}^{*} \rightarrow \mathrm{SM}$ also washes-out the asymmetries, but is suppressed for low $\left.T_{\mathrm{EW}}\right)$. If these processes are in equilibrium after the EW phase transition, they will very rapidly drive

$$
\Delta_{\alpha} \rightarrow \sum_{\beta \neq \alpha} \Delta_{\beta}
$$

which, together with (A.11), leads to $\Delta_{\alpha} \rightarrow 0$. While it is possible for the decay process $X_{\alpha} \rightarrow X_{\beta}+$ SM to be suppressed if the different species are quite degenerate, this automatically results in unsuppressed kinetic equilibrium. This seems to rule-out asymmetric production as a viable mechanism to avoid complete wash-out of the amount of light dark matter non-thermally produced during the EW phase transition, the reason being that it is not possible (at least in this simple scenario) to keep all the processes that tend to erase the asymmetries $\Delta_{\alpha}$ out of equilibrium after the EW phase transition, while having an efficient particle production at the transition itself.

Open Access. This article is distributed under the terms of the Creative Commons Attribution License which permits any use, distribution and reproduction in any medium, provided the original author(s) and source are credited.

\section{References}

[1] WMAP collaboration, E. Komatsu et al., Seven-Year Wilkinson Microwave Anisotropy Probe (WMAP) Observations: Cosmological Interpretation, Astrophys. J. Suppl. 192 (2011) 18 [arXiv:1001.4538] [INSPIRE].

[2] S. Dodelson and L.M. Widrow, Sterile-neutrinos as dark matter, Phys. Rev. Lett. 72 (1994) 17 [hep-ph/9303287] [INSPIRE].

[3] J. Preskill, M.B. Wise and F. Wilczek, Cosmology of the Invisible Axion, Phys. Lett. B 120 (1983) 127 [INSPIRE].

[4] L. Abbott and P. Sikivie, A Cosmological Bound on the Invisible Axion, Phys. Lett. B 120 (1983) 133 [InSPIRE].

[5] M. Dine and W. Fischler, The Not So Harmless Axion, Phys. Lett. B 120 (1983) 137 [INSPIRE].

[6] T. Moroi and L. Randall, Wino cold dark matter from anomaly mediated SUSY breaking, Nucl. Phys. B 570 (2000) 455 [hep-ph/9906527] [InSPIRE].

[7] D.J. Chung, E.W. Kolb and A. Riotto, Nonthermal supermassive dark matter, Phys. Rev. Lett. 81 (1998) 4048 [hep-ph/9805473] [INSPIRE].

[8] D.J. Chung, E.W. Kolb and A. Riotto, Superheavy dark matter, Phys. Rev. D 59 (1999) 023501 [hep-ph/9802238] [InSPIRE].

[9] D.J. Chung, E.W. Kolb and A. Riotto, Production of massive particles during reheating, Phys. Rev. D 60 (1999) 063504 [hep-ph/9809453] [INSPIRE].

[10] E.W. Kolb, D.J. Chung and A. Riotto, WIMPzillas!, hep-ph/9810361 [INSPIRE].

[11] T. Konstandin and G. Servant, Cosmological Consequences of Nearly Conformal Dynamics at the TeV scale, JCAP 12 (2011) 009 [arXiv:1104.4791] [INSPIRE]. 
[12] S. Hawking, I. Moss and J. Stewart, Bubble Collisions in the Very Early Universe, Phys. Rev. D 26 (1982) 2681 [INSPIRE].

[13] R. Watkins and L.M. Widrow, Aspects of reheating in first order inflation, Nucl. Phys. B 374 (1992) 446 [inSPIRE].

[14] T. Konstandin and G. Servant, Natural Cold Baryogenesis from Strongly Interacting Electroweak Symmetry Breaking, JCAP 07 (2011) 024 [arXiv:1104.4793] [INSPIRE].

[15] S.Y. Khlebnikov, Fluctuation - dissipation formula for bubble wall velocity, Phys. Rev. D 46 (1992) 3223 [inSPIRE].

[16] P.B. Arnold, One loop fluctuation - dissipation formula for bubble wall velocity, Phys. Rev. D 48 (1993) 1539 [hep-ph/9302258] [INSPIRE].

[17] G.D. Moore and T. Prokopec, How fast can the wall move? A Study of the electroweak phase transition dynamics, Phys. Rev. D 52 (1995) 7182 [hep-ph/9506475] [INSPIRE].

[18] G.D. Moore, Electroweak bubble wall friction: Analytic results, JHEP 03 (2000) 006 [hep-ph/0001274] [INSPIRE].

[19] P. John and M. Schmidt, Do stops slow down electroweak bubble walls?, Nucl. Phys. B 598 (2001) 291 [Erratum ibid. B 648 (2003) 449] [hep-ph/0002050] [INSPIRE].

[20] P.J. Steinhardt, Relativistic detonation waves and bubble growth in false vacuum decay, Phys. Rev. D 25 (1982) 2074 [inSPIRE].

[21] M. Gyulassy, K. Kajantie, H. Kurki-Suonio and L.D. McLerran, Deflagrations and Detonations as a Mechanism of Hadron Bubble Growth in Supercooled Quark Gluon Plasma, Nucl. Phys. B 237 (1984) 477 [inSPIRE].

[22] H. Kurki-Suonio, Deflagration bubbles in the quark - hadron phase transition, Nucl. Phys. B 255 (1985) 231 [inSPIRE].

[23] K. Kajantie and H. Kurki-Suonio, Bubble Growth and Droplet Decay in the Quark Hadron Phase Transition in the Early Universe, Phys. Rev. D 34 (1986) 1719 [InSPIRE].

[24] M. Laine, Bubble growth as a detonation, Phys. Rev. D 49 (1994) 3847 [hep-ph/9309242] [INSPIRE].

[25] H. Kurki-Suonio and M. Laine, Supersonic deflagrations in cosmological phase transitions, Phys. Rev. D 51 (1995) 5431 [hep-ph/9501216] [INSPIRE].

[26] J.R. Espinosa, T. Konstandin, J.M. No and G. Servant, Energy Budget of Cosmological First-order Phase Transitions, JCAP 06 (2010) 028 [arXiv: 1004.4187] [INSPIRE].

[27] M. Kamionkowski, A. Kosowsky and M.S. Turner, Gravitational radiation from first order phase transitions, Phys. Rev. D 49 (1994) 2837 [astro-ph/9310044] [INSPIRE].

[28] D. Bödeker and G.D. Moore, Can electroweak bubble walls run away?, JCAP 05 (2009) 009 [arXiv: 0903.4099] [INSPIRE].

[29] T. Konstandin and J.M. No, Hydrodynamic obstruction to bubble expansion, JCAP 02 (2011) 008 [arXiv: 1011.3735] [INSPIRE].

[30] C. Hogan, Nucleation of cosmological phase transitions, Phys. Lett. B 133 (1983) 172 [INSPIRE]. 
[31] J. Giblin, John T., L. Hui, E.A. Lim and I.-S. Yang, How to Run Through Walls: Dynamics of Bubble and Soliton Collisions, Phys. Rev. D 82 (2010) 045019 [arXiv:1005.3493] [INSPIRE].

[32] Joy Division, Unknown Pleasures, Factory Records (1979).

[33] XENON100 collaboration, E. Aprile et al., Dark Matter Results from 225 Live Days of XENON100 Data, Phys. Rev. Lett. 109 (2012) 181301 [arXiv:1207.5988] [INSPIRE].

[34] O. Lebedev, H.M. Lee and Y. Mambrini, Vector Higgs-portal dark matter and the invisible Higgs, Phys. Lett. B 707 (2012) 570 [arXiv:1111.4482] [INSPIRE].

[35] A. Djouadi, O. Lebedev, Y. Mambrini and J. Quevillon, Implications of LHC searches for Higgs-portal dark matter, Phys. Lett. B 709 (2012) 65 [arXiv:1112.3299] [INSPIRE].

[36] T. Hambye and M.H. Tytgat, Confined hidden vector dark matter, Phys. Lett. B 683 (2010) 39 [arXiv:0907.1007] [INSPIRE].

[37] S. Kanemura, S. Matsumoto, T. Nabeshima and N. Okada, Can WIMP Dark Matter overcome the Nightmare Scenario?, Phys. Rev. D 82 (2010) 055026 [arXiv:1005.5651] [INSPIRE].

[38] R. Young and A. Thomas, Octet baryon masses and sigma terms from an $\mathrm{SU}(3)$ chiral extrapolation, Phys. Rev. D 81 (2010) 014503 [arXiv:0901.3310] [INSPIRE].

[39] J. Martin Camalich, L. Geng and M. Vicente Vacas, The lowest-lying baryon masses in covariant SU(3)-flavor chiral perturbation theory, Phys. Rev. D 82 (2010) 074504 [arXiv: 1003.1929] [INSPIRE]. 